\title{
Matrix Effective Theories of the Fractional Quantum Hall effect
}

\author{
Andrea Cappelli and Ivan D. Rodriguez \\ I.N.F.N. and Dipartimento di Fisica \\ Via G. Sansone 1, 50019 Sesto Fiorentino - Firenze, Italy \\ E-mail: andrea.cappelli@fi.infn.it, rodriguez@fi.infn.it
}

\begin{abstract}
The present understanding of nonperturbative ground states in the fractional quantum Hall effect is based on effective theories of the Jain "composite fermion" excitations. We review the approach based on matrix variables, i.e. D0 branes, originally introduced by Susskind and Polychronakos. We show that the Maxwell-Chern-Simons matrix gauge theory provides a matrix generalization of the quantum Hall effect, where the composite-fermion construction naturally follows from gauge invariance. The matrix ground states obtained by suitable projections of higher Landau levels are found to be in one-to-one correspondence with the Laughlin and Jain hierarchical states. The matrix theory possesses a physical limit for commuting matrices that could be reachable while staying in the same phase.
\end{abstract}




\section{Introduction}

The quantized Hall effect occurs in systems of planar electrons inside layered semiconductors, that are placed in strong magnetic fields $\mathbf{B}(\sim 10$ Tesla $)$ and very low temperatures $(T \sim 1 \mathrm{mK}-1 \mathrm{~K})[1]$. For certain values of the field, the longitudinal Ohmic current vanishes and the transverse component $R_{x y}$ of the resistivity (Hall resistivity) is quantized (Fig.1):

$$
\begin{aligned}
& R_{x y}=\sigma_{x y}^{-1}=\nu^{-1} \frac{h}{e^{2}}, \quad \nu=1,2,3, \ldots, \frac{1}{3}, \frac{1}{5}, \ldots, \frac{2}{5}, \frac{2}{7}, \frac{3}{5}, \ldots, \\
& R_{x x}=\sigma_{x x}=0,
\end{aligned}
$$

where $\nu$ is the "filling fraction", that can be integer or fractional [2]. The regimes in which the values of the resistivity are given by (1) are called "plateaux" of the Quantum Hall effect (QHE). They correspond to very stable gapful ground states with uniform density $\bar{\rho}=\nu e \mathbf{B} / h c$, where the electrons behaves like a fluid with characteristic quantum effects [1]. The low-energy excitations are local deformations in the density (vortices) called quasi-holes and quasi-particles; the density waves are also gapful such that the quantum fluid is incompressible. The integer Hall effect can be described in terms of free electrons filling up the Landau levels, while the fractional effect requires to consider interacting electrons.

In 1983, Laughlin proposed a phenomenological theory for the fillings $\nu=1 /(2 k+1)$, with $k$ positive integer [3]: he described the incompressible fluid and predicted quasiparticles with fractional charge that were observed in 1997 [4]. Other filling fractions not described by Laughlin's theory are observed experimentally, belonging to the series $\nu=n /(2 n k \pm 1)$, where $n>1$ and $k$ are positive integers [1]. Upon introducing the idea of "composite fermions" excitations, Jain argued that these fractional quantum Hall states actually correspond to integer quantum Hall states of composite fermions [5]. Based on this relation, Jain obtained trial wavefunctions that are confirmed by the numerical analyses. Moreover, weakly-interacting composite-fermion excitations have been observed in several experiments [1]. Fradkin and Lopez [6] and others [7] realized the Jain correspondence in quantum field theory by letting the electrons to interact with a "statistical" Chern-Simons gauge field. They studied the theory within the mean field approximation and reproduced the Jain ground states and some of their phenomenological features.

In this contribution, we review another possible effective theory for the fractional QHE, that is based on matrix models or, more precisely, on gauge theories of matrices in $(2+1)$ dimensions, that are equivalent to noncommutative gauge theories. This approach is not yet fully developed, but it presents some interesting features that we believe are worth discussing.

The presentation is organized as follows: the next section contains a short introduction to the phenomenology of the integer and fractional QHE. We review the Laughlin theory [3], the Jain interpretation of the fractional QHE [5] and the field theory proposed by Fradkin and Lopez [6]. The third section deals with the Chern-Simons 


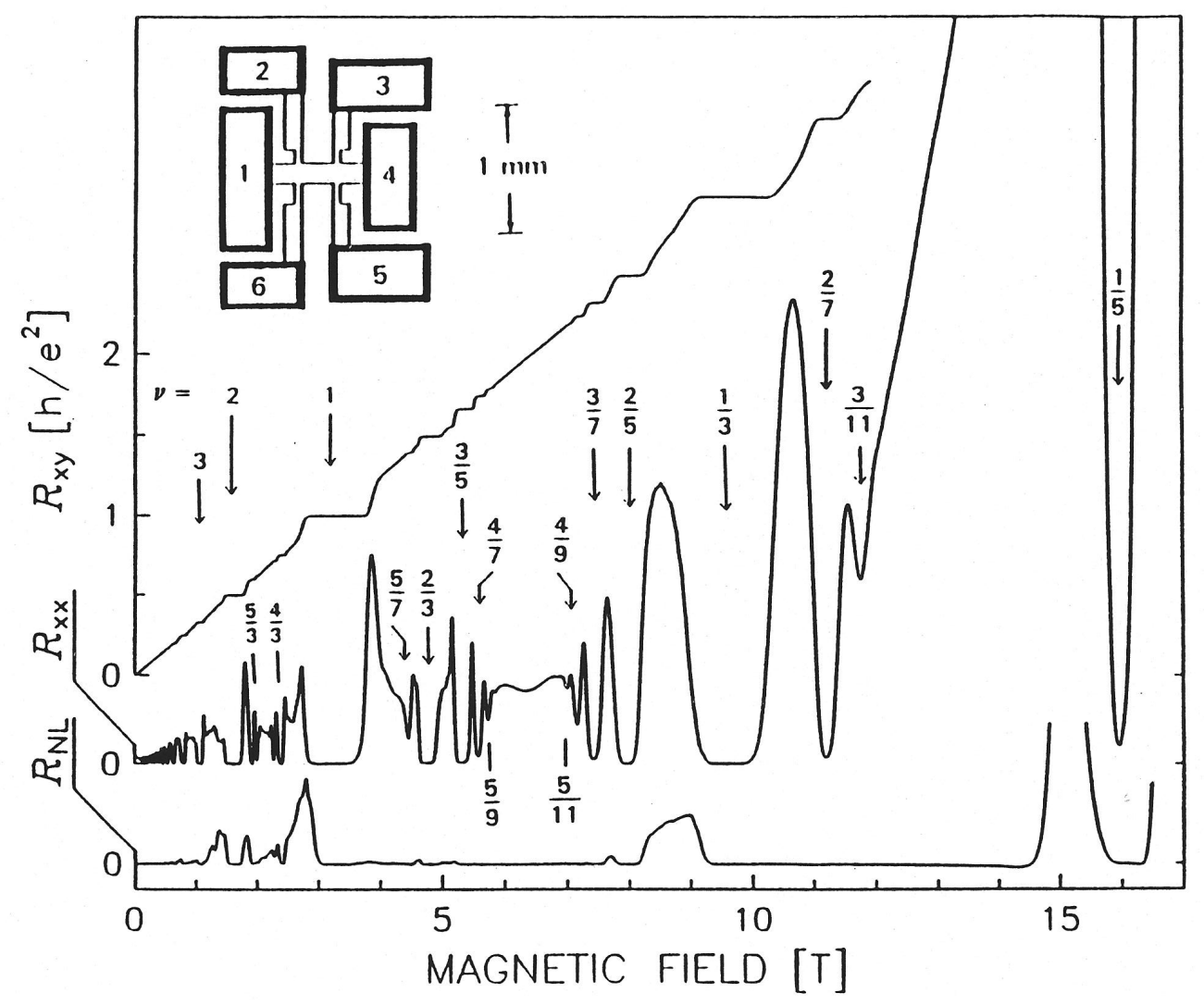

Figure 1. Diagonal and transverse resistivities in the fractional quantum Hall effect $[5]$.

matrix model, and reviews the work by Susskind and Polychronakos. Using results for D0-branes in string theory, Susskind showed that two-dimensional semiclassical incompressible fluids in strong magnetic field could be described by the noncommutative Chern-Simons theory [8]. Indeed, the use of noncommuting spatial coordinates, $x_{1}, x_{2}$, i.e. $\left[x_{1}, x_{2}\right]=i \theta$, implies a generalized uncertainty relation that controls the effective size of electrons and thus reduces the density of the fluid, leading to $\nu=1 /(1+\mathbf{B} \theta)<1$. Afterwards, Polychronakos modified the theory to describe a finite droplet of fluid, and obtained the $U(N)$ matrix gauge theory called Chern-Simons matrix model [9]. From the quantization of this theory, one finds the important result that the ground states are exactly given by the Laughlin wave functions [10][11]. However, the Chern-Simons matrix model does not naturally describe the more general Jain states and the full quantum theory does not reproduce the electron system of the QHE [12][13].

Section 4 is devoted to our proposal of the Maxwell-Chern-Simons matrix theory [12]: this is a generalization of Susskind-Polychronakos theory that contains an additional coupling $g \geq 0$ controlling matrix noncommutativity. At $g=0$, the theory corresponds to a matrix generalization of the Landau levels, with an exponentially growing density of states that is typical of matrix theories. We introduce a set of projections that not only limit the degeneracy but also uniquely selects ground states 
that are matrix analogs of the expected Laughlin and Jain states (section 5). This is the most interesting feature of the matrix approach to the QHE, namely that the phenomenological ground states arise naturally from gauge invariance and the projections. The same ground states are also found in the semiclassical analysis of the theory [14]: they correspond to incompressible fluids with piecewise constant density, as expected [5]. In section 6, we discuss the Maxwell-Chern-Simons matrix theory for $g>0$ : in the $g=\infty$ limit, the matrix coordinates commute and the theory describes ordinary electrons in Landau levels with $O\left(1 / r^{2}\right)$ interaction, which is a good approximation of the QHE system [1]. Although the phase diagram $(0<g<\infty)$ of the Maxwell-ChernSimons theory is not yet known, we conjecture that the matrix ground states found at $g=0$ have a smooth $g \rightarrow \infty$ limit into the phenomenological Laughlin and Jain states (no phase transition for finite $g$ values) [12]. The proof of this fact would confirm the physical relevance of the matrix theory approach to the fractional QHE. In the conclusions (section 7), we discuss some developments of this line of research.

This paper is dedicated to the vivid memory of Alyosha Zamolodchikov.

\section{Review of the Fractional Quantum Hall Effect}

\subsection{Landau levels}

Consider planar electrons of mass $m$ and electric charge $e$ in an uniform magnetic field $\mathbf{B}$ (in units $\hbar=1, c=1$ ). The one-particle Hamiltonian is given by:

$$
H=-\frac{1}{2 m}(\nabla-i e \mathbf{A})^{2} \text {. }
$$

We work in the symmetric gauge for the vector potential, $A_{i}=(\mathbf{B} / 2) \epsilon_{i j} x^{j}, i, j=1,2$. The magnetic field introduces a length scale, the so-called magnetic length, $\ell=\sqrt{2 / e \mathbf{B}}$. The use of holomorphic spatial coordinates $z=x_{1}+i x_{2}$ and $\bar{z}=x_{1}-i x_{2}$, is natural in the QHE [15]. By introducing two commuting sets of harmonic oscillators $\left(\partial=\frac{\partial}{\partial z}\right.$ and $\left.\bar{\partial}=\frac{\partial}{\partial \bar{z}}\right)$,

$$
\begin{aligned}
d=\frac{z}{2 \ell}+\ell \bar{\partial}, & d^{\dagger}=\frac{\bar{z}}{2 \ell}-\ell \partial, & & {\left[d, d^{\dagger}\right]=1, } \\
c=\frac{\bar{z}}{2 \ell}+\ell \partial, & c^{\dagger}=\frac{z}{2 \ell}-\ell \bar{\partial}, & & {\left[c, c^{\dagger}\right]=1, }
\end{aligned}
$$

the Hamiltonian (2) and the angular momentum can be written as follows:

$$
\begin{aligned}
& H=\omega\left(d^{\dagger} d+\frac{1}{2}\right), \\
& J=c^{\dagger} c-d^{\dagger} d,
\end{aligned}
$$

where $\omega=e \mathbf{B} / m$ is the cyclotron frequency. Since the operators $c$ and $d$ commute, the spectrum consists of infinitely degenerate levels ( $c^{\dagger} c$ excitations) with energies $\epsilon_{n}=\omega n$, i.e. the Landau levels ( $d^{\dagger} d$ ladder). The degenerate states correspond to the semiclassical cyclotron orbits, that are quantized by the condition that the contained flux is a multiple 
of the quantum unit $\phi_{0}=e h / c$, i.e. $B A_{j}=j \phi_{0}$, with $j$ the angular momentum eigenvalue. In the lowest Landau level, the one-particle wave functions take the form:

$$
\varphi_{j}(z, \bar{z})=\frac{1}{\ell \sqrt{\pi}} \frac{1}{j !}\left(\frac{z}{\ell}\right)^{j} e^{-z \bar{z} /\left(2 \ell^{2}\right)}, \quad d \varphi_{j}(z, \bar{z})=0
$$

i.e. they are holomorphic in $z$ up to an exponential factor. The associated one-particle densities are indeed peaked at the semiclassical orbits.

On a finite region of area $A$, the number of degenerate states is equal to the flux through the system in quantum units, $N_{\phi}=\mathbf{B} A / \phi_{0}$. In completely filled Landau levels, the Hall conductivity is given by $\sigma_{x y}=R_{x y}^{-1}=\nu e^{2} / h$, where $\nu=N / N_{\phi}$ is the filling fraction, i.e. the number of electrons $N$ divided by the number of available states. Figure 2 (a) shows the $\nu=n$ case in which $n$ levels are filled with one electron per orbital (the spin degree of freedom is frozen in the direction of $\mathbf{B}$ ). The density is uniform and the electron fluid is incompressible due to the exclusion principle, the gap being given by $\omega$. Thus the simple theory of free electrons in Landau levels is sufficient to describe the main physical properties of the integer QHE. (The formation of the plateaux near integer fillings is explained by the localization of excitations due to disorder) [1].

On the other hand, if there are many empty orbitals like in the case $\nu=1 / 3$ (Fig.2(b)), the free-electron states are compressible, in contrast with the experimental observation: the fractional QHE requires the study of interacting electrons. The formation of the gap by the Coulomb potential is clearly nonperturbative: one should try an approach based on ansatzs and effective theories, supplemented by numerical analyses. It turns out that the ground states are condensates of charges and vortices which have some analogies with superfluids and confined gauge theories, but are also specific of the two-dimensional parity breaking setting.

\subsection{The Laughlin theory}

In a remarkable paper [3], Laughlin proposed a class of trial wave functions given by:

$$
\Psi_{m}\left(z_{1}, z_{2}, \ldots, z_{N}\right)=\prod_{i<j=1}^{N}\left(z_{i}-z_{j}\right)^{m} e^{-\frac{1}{2} \sum_{i}^{N}\left|z_{i}\right|^{2}},
$$

with $N$ the number of electrons and $m$ an integer parameter. Hereafter we set the magnetic length $\ell=1$. The wave functions (6) describe spinless electrons in the lowest Landau level: $m$ must be odd, $m=2 k+1$, for antisymmetry of fermions. In order to determine the properties of these states, Laughlin used the analogy with a twodimensional plasma, as it follows. The determination of the one-particle density can be reduced to the analysis of the two-dimensional statistical model of charges defined by,

$$
\begin{aligned}
& Z_{\text {plasma }}=\left\|\Psi_{m}\right\|^{2}=\int \prod_{i=1}^{N} d^{2} z_{i} e^{-\beta H_{\text {plasma }}}, \\
& H_{\text {plasma }}=m \sum_{i=1}^{N}\left|z_{i}\right|^{2}-m^{2} \sum_{i<j=1}^{N} \log \left|z_{i}-z_{j}\right|^{2} .
\end{aligned}
$$


(a)

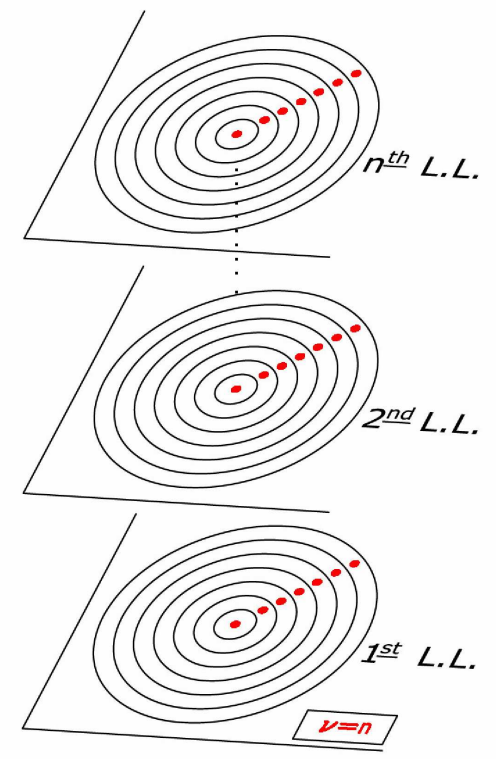

(b)

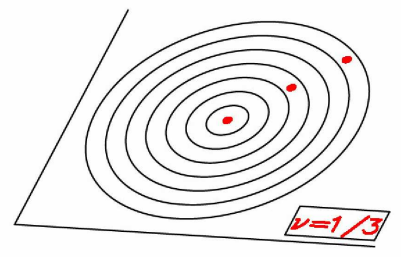

Figure 2. Graphical representation of the Landau levels: (a) integer and (b) fractional filling.

In this equation, $H_{\text {plasma }}$ describes a classical plasma of charges in a uniform background at temperature $\beta=1 / \mathrm{m}$. Knowing that this plasma is totally screened for small values of $m$, Laughlin could argue that the density is uniform and could calculate the gap of excitations. For constant density, the parameter $m$ can be readily related to the filling fraction by $\nu=1 / m$. Note that Laughlin's wavefunctions vanishes as $\left(z_{i}-z_{j}\right)^{m}$ when any two particles $i$ and $j$ approach each other: namely, the amplitude for nearby particles is very small and the expectation value of the Coulomb energy is consequently reduced. This is a rather successful property from the variational point of view, since other wave functions with same average density do have this feature. Numerical experiments show that the Laughlin wavefunction is actually very close to the exact ground state for several short-range repulsive interactions [1][3][16].

Laughlin also proposed the wave functions of the low-energy quasiholes excitations: they are localized density deformations,

$$
\Psi_{q h}=\left(\eta ; z_{1}, \ldots, z_{N}\right)=\prod_{i=1}^{N}\left(\eta-z_{i}\right) \prod_{i<j=1}^{N}\left(z_{i}-z_{j}\right)^{2 k+1} e^{-\frac{1}{2 \ell^{2}} \sum_{i}\left|z_{i}\right|^{2}},
$$

with $\eta$ the position of the vortex (Fig.3). To calculate the charge of the quasi-hole, one can use the plasma analogy (7):

$$
\left\|\Psi_{q h}\right\|^{2}=\int \prod_{i=1}^{N} d^{2} z_{i} e^{-\beta\left(m \sum_{i}\left|z_{i}\right|^{2}-m^{2} \sum_{i<j} \log \left|z_{i}-z_{j}\right|^{2}-m \sum_{i} \log \left|z_{i}-\eta\right|\right)} .
$$

Comparing (9) with (7), we observe that the electrons feel the presence of a charge $1 / \mathrm{m}$ at the point $z=\eta$ : thus, the quasi-holes have fractional charge $Q_{q h}=e / m[3]$. 


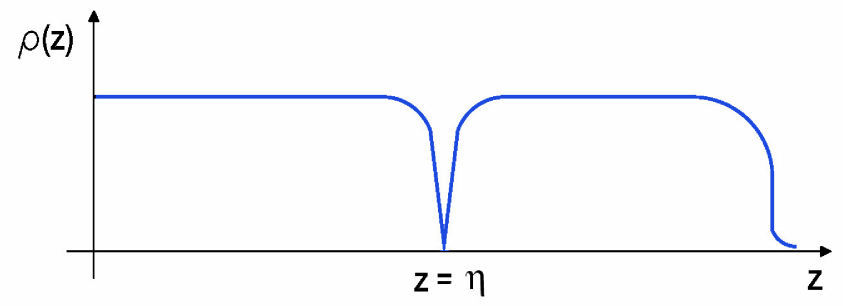

Figure 3. Schematic plot of the density of the electron fluid in presence of a quasi-hole at $z=\eta$.

In the wave function for two quasi-holes,

$$
\Psi_{2 q h}\left(\eta_{1}, \eta_{2} ; z_{1}, \ldots, z_{N}\right)=\left(\eta_{1}-\eta_{2}\right)^{\frac{1}{2 k+1}} \prod_{i}\left(\eta_{1}-z_{i}\right) \prod_{i}\left(\eta_{2}-z_{i}\right) \Psi_{m}
$$

Laughlin introduced the term $\left(\eta_{1}-\eta_{2}\right)$ raised to a fractional power, that is necessary for charge equilibration in the plasma (9); he assumed a holomorphic dependence as for the electron coordinates [17]. If we rotate one quasi-hole around the other, we obtain:

$$
\Psi_{2 q h}\left(\left(\eta_{1}-\eta_{2}\right) \rightarrow e^{i \pi}\left(\eta_{1}-\eta_{2}\right)\right)=e^{i \frac{\pi}{2 k+1}} \Psi_{2 q h}\left(\eta_{1}, \eta_{2}\right) .
$$

Therefore, the wave function acquires a non-trivial phase under exchanges of excitations: the quasi-holes have "fractional statistics", $\frac{\theta}{\pi}=\frac{1}{2 k+1}$. This is a long-range, topological interaction of vortices, that is allowed in parity breaking two-dimensional systems [17]. The fractional charge and statistics of excitations are confirmed by the effective field theory descriptions to be discussed later. The fractional charge has been observed in experiments of quasiparticle tunneling [4]; the fractional statistic has not been detected directly but there are indirect confirmations [1].

\subsection{The Jain interpretation of the fractional quantum Hall effect}

In Fig. 1, one finds stable plateaux at other filling fractions that are nicely accounted by the series $\nu=n /(2 n k \pm 1)$ with $n>1$. The phenomenological theory due to Jain explains them as follows: the argument starts by observing that the inverse filling,

$$
\frac{1}{\nu}=\frac{N_{\phi}}{N}= \pm \frac{1}{n}+2 k
$$

is equal the number of fluxes per electron. Imagine that it is possible to bound an even number of fluxes, i.e. $2 k$, to each electron, to form a new quasiparticle called "composite fermion". (Note that an even number of flux quanta yield an integer Aharonov-Bohm phase and keeps the fermionic statistics). When $2 k$ fluxes are attached to each electron, the same number of fluxes are removed from the external magnetic field: therefore, the filling fraction of the system of composite fermions is given by

$$
\frac{1}{\nu^{*}}=\frac{N_{\Phi}-2 k N}{N}=\frac{1}{\nu}-2 k= \pm \frac{1}{n}, \quad \mathbf{B}^{\star}=\mathbf{B}-2 k 2 \pi \rho_{0},
$$


corresponding to an integer QHE. The reduced magnetic field felt by the new particles is given by $\mathbf{B}^{\star}$. This is indeed observed: many experiments confirm the existence of weakly interacting excitations feeling the reduced magnetic field, i.e. behaving as Jain's composite fermions [1]. The incompressibility of the fractional QHE is explained by Jain as due to the equivalence between the system of electrons with $\nu=n /(2 n k+1)$ and the integer QHE of composite fermions with $\nu^{\star}=n$.

Following the Jain argument, the flux attachment is clear in the form of the Laughlin wave function $\left(\nu^{\star}=1\right)$ : the factor $\Pi_{i<j}^{N}\left(z_{i}-z_{j}\right)^{2 k}$ yields Aharonov-Bohm phase of $2 k$ flux quanta to any electron, and the rest is the wave function of the filled first Landau level. In the general case of $\nu=n /(2 n k+1)$, the ground state wave functions proposed by Jain on the basis of his equivalence are:

$$
\Psi_{2 k+\frac{1}{n}}\left(z_{1}, \ldots, z_{N}\right)=\prod_{i<j}^{N}\left(z_{i}-z_{j}\right)^{2 k} \Psi_{\frac{1}{n}}\left(z_{1}, \ldots, z_{N}\right),
$$

with $\Psi_{1 / n}\left(z_{1}, \ldots, z_{N}\right)$ being the wave functions with $n$ completely filled levels (Slater determinants). The Jain wave functions (14) have been confirmed by comparison with numerical results of exact diagonalization of the Hamiltonian with Coulomb interaction [5]. The fillings $\nu=n /(2 n k-1)$ are also described by (14) with charge-conjugate term $\Psi_{1 / n} \rightarrow \bar{\Psi}_{1 / n}$.

The Jain scheme also provide excellent approximations for the quasi-holes and quasi-particles excitations [5]. For instance, a quasi-particle in the origin for the Jain state with $\nu=n /(2 n k+1)$ is given by,

$$
\Psi_{q p ; 2 k+\frac{1}{n}}\left(z_{1}, \ldots, z_{N}\right)=\prod_{i<j}^{N}\left(z_{i}-z_{j}\right)^{2 k} \Psi_{q p ; \frac{1}{n}}\left(z_{1}, \ldots, z_{N}\right),
$$

where $\Psi_{q p ; 1 / n}\left(z_{1}, \ldots, z_{N}\right)$ corresponds to the wave function of $n$ filled Landau levels and one electron in the first orbital of the $(n+1)$-th Landau level. The corresponding localized density has an excess of charge at the origin of the droplet.

\subsection{Fermion Chern-Simons field theory}

Among the effective field theories that have been proposed to describe the fractional QHE, we recall the theory of non-relativistic fermions coupled to the Abelian ChernSimons "statistical" interaction, that has been developed by Fradkin and Lopez [6] and others [7]. The action can be schematically written:

$$
S=\frac{\kappa}{4 \pi} \int \varepsilon^{\mu \nu \rho} a_{\mu} \partial_{\nu} a_{\rho}+\int J_{\mu} a_{\mu}+S_{\text {fermion }} .
$$

Consider the Gauss law of this theory:

$$
j_{0}(\vec{x})=-\frac{\kappa}{2 \pi} \mathcal{B}(\vec{x})=-\frac{\kappa}{2 \pi} \epsilon^{i j} \partial_{i} a_{j}(\vec{x}),
$$

where $\mathcal{B}$ is the "statistical" magnetic field and $\kappa$ the Chern-Simons coupling constant. At quantum level, this is an operator constraint which selects the physical space of states. These are charge-flux composites: every particle with unit electric charge carries 
a magnetic charge equal to $2 \pi / \kappa$. The wave functions for these composite particles exhibits Aharonov-Bohm effects changing the statistics. Therefore a fermion coupled to a Chern-Simons gauge field behaves like an anyon with statistical angle $\theta / \pi=1 / \kappa$, measured with respect to the Fermi statistics [6]. If $\kappa=1 / 2 k$, where $k$ is an integer, then $\theta / \pi=2 k$ and the composite states are still fermions.

A ground state with uniform density $\left\langle j_{0}(z)\right\rangle=\bar{\rho}$ implies a constant field:

$$
\langle\mathcal{B}\rangle=-\frac{2 \pi \bar{\rho}}{\kappa}=-2 k 2 \pi \rho_{0}, \quad\langle\mathcal{E}\rangle=0 .
$$

Eq. (18) shows that, within the mean field approximation, the effect of the statistical gauge field is to change the effective flux experienced by the fermionic excitations. The effective magnetic field is $B_{\text {eff }}=\mathbf{B}+\langle\mathcal{B}\rangle=\mathbf{B}-2 \kappa 2 \pi \rho_{0}$, in agreement with Jain's argument (13).

The uniform effective magnetic field $B_{\text {eff }}$ defines a new set of effective Landau levels. Each level has a degeneracy equal to the total number of effective flux quanta $N_{e f f}$ and the separation between levels is the effective cyclotron frequency $\omega_{\text {eff }}=\left|B_{\text {eff }}\right| / \mathrm{m}$. Similarly, there is an effective cyclotron radius $\ell_{\text {eff }}$. It is easy to see that the mean-field approximation (uniform density) (18) is selfconsistent only if the fermions fill exactly an integer number $n$ of effective Landau levels. This reproduces Jain's theory: the fractional QHE is the integer QHE of a system of electrons dressed by an even number of flux quanta. The allowed filling fractions are those obtained by Jain: $\nu=n /(2 n k \pm 1)$ [6]. Further results of this approach are reviewed in [18].

Let us mention for completeness the effective field theory approaches based on $(1+1)$ dimensional conformal field theories. As originally observed by Wen [19], a droplet of incompressible fluid possess low-energy massless chiral excitations at the edge, that can be described by conformal field theories with $U(1)$ current, also called chiral

Luttinger liquid theories, and their generalizations. These theories of the QHE have been extensively developed in the last 20 years and can describe the low-energy physics occurring in conduction experiments [1] [19] [20]. In this approach, the formation of the incompressible fluid is assumed and cannot be derived, since the dimensional reduction is only possible for these specific states; actually, there is a different conformal field theory for each plateaux, whose form can be inferred by the properties of excitations and other data. In the following, we deal with $(2+1)$-dimensional effective theories that could explain the formation of incompressible ground states.

\section{Semiclassical incompressible fluid and noncommutative Chern-Simons theory}

In this section we introduce the effective theories of the fractional QHE based on matrix degrees of freedom, equivalent to noncommutative field theories. The subject was initiated by Susskind in 2001, who observed the analogies between the QHE and the physics of D-branes in string theory [8][21]. We shall find that the matrix d.o.f. have associated a gauge field and their Gauss law provides another realization of Jain's flux 
attachment to particles (13). The description of the QHE by matrix theories is far less developed than the fermion Chern-Simons theory, but there are some nice features like the explicit relation with wave functions; in our opinion, the matrix theories could provide another view on the physics of composite fermions.

We start by reviewing Susskind's work [8], who observed that the semiclassical limit of noncommutative Chern-Simons field theory could describe incompressible fluids in high magnetic field with Laughlin's filling fractions [8]. Consider $N$ first-quantized electrons with two-dimensional coordinates $X_{\alpha}^{a}(t), a=1,2, \alpha=1, \ldots, N$, placed in a strong magnetic field $\mathbf{B}$, such that their action can be projected to the lowest Landau level [22],

$$
L=\frac{e \mathbf{B}}{2} \sum_{\alpha=1}^{N} \epsilon_{a b} X_{\alpha}^{a} \dot{X}_{\alpha}^{b}
$$

We now consider the limit of the particle forming a continuous fluid:

$$
\vec{X}_{\alpha}(t) \rightarrow \vec{X}(\vec{x}, t), \quad \vec{X}(\vec{x}, t=0)=\vec{x},
$$

where $\vec{x}$ are the coordinates of an initial, reference configuration of the fluid. The resulting fluid mechanics is in the Lagrangian formulation, because the field $\vec{X}(\vec{x})$ follows the motion of the fluid [23]. For incompressible fluids, the constraint of constant density, $\rho(\vec{X})=\rho_{o}$, can be written in terms of Poisson brackets $\{\cdot, \cdot\}$ of the $\vec{x}$ coordinate as follows:

$$
\rho_{o}=\rho(\vec{x})=\rho_{o}\left|\frac{\partial \vec{X}}{\partial \vec{x}}\right|=\frac{\rho_{o}}{2} \epsilon_{a b}\left\{X^{a}, X^{b}\right\} .
$$

This constraint can be added to the Lagrangian by using the Lagrange multiplier $A_{0}$,

$$
L=\frac{e \mathbf{B} \rho_{\mathbf{o}}}{2} \int d^{2} x\left[\epsilon_{a b} X^{a}\left(\dot{X}^{b}-\theta\left\{X^{b}, A_{0}\right\}\right)+2 \theta A_{0}\right] ;
$$

in this equation, we introduced the constant $\theta$,

$$
\theta=\frac{1}{2 \pi \rho_{o}},
$$

that will later parameterize the non-commutativity.

The Lagrangian (22) is left invariant by reparametrizations of the $\vec{x}$ variable with unit Jacobian, the area-preserving diffeomorphism, also called $w_{\infty}$ transformations [15]: they correspond to changes of the original labels of the fluid at $t=0$ (cf. Eq.(20)) [8][23]. The $w_{\infty}$ symmetry can be put into the form of a gauge invariance by introducing the gauge potential $\vec{A}$, as follows:

$$
X^{a}=x^{a}+\theta \epsilon_{a b} A_{b}(x),
$$

and by rewriting the Lagrangian (22) in the Chern-Simons form:

$$
L=-\frac{k}{4 \pi} \int d^{2} x \epsilon_{\mu \nu \rho}\left(\partial_{\mu} A_{\nu} A_{\rho}+\frac{\theta}{3}\left\{A_{\mu}, A_{\nu}\right\} A_{\rho}\right),
$$


where $A_{\mu}=\left(A_{0}, A_{a}\right)$ is the three-dimensional gauge field. The coupling constant $k$ parameterizes the filling fraction of the semiclassical fluid:

$$
\nu^{(c l)}=\frac{2 \pi \rho_{o}}{e \mathbf{B}}=\frac{1}{e \mathbf{B} \theta}=\frac{1}{k} .
$$

Based on the result (25), Susskind conjectured that the non-commutative (Abelian) Chern-Simons theory could be the complete theory going beyond the continuous fluid approximation, i.e. accounting for the granularity of the electrons. Its action is [24]:

$$
L_{N C C S}=-\frac{k}{4 \pi} \int d^{2} x \epsilon_{\mu \nu \rho}\left(\partial_{\mu} A_{\nu} \star A_{\rho}-\frac{2 i}{3} A_{\mu} \star A_{\nu} \star A_{\rho}\right),
$$

involving the Moyal star product:

$$
\left.(F \star G)\left(x_{1}, x_{2}\right)=F\left(x_{1}, x_{2}\right) e^{\frac{i \theta}{2}} \overleftarrow{\partial_{x_{1}}} \overrightarrow{\partial_{x_{2}}}-\overleftarrow{\partial_{x_{2}}} \overrightarrow{\partial_{x_{1}}}\right) G\left(x_{1}, x_{2}\right) .
$$

Actually, the two Lagrangians (27) and (25) agree to leading order in $\theta$, i.e. for dense fluids. In the new Lagrangian (27), the gauge fields with Moyal product have become Wigner functions (see next section) of the noncommuting operators, $\widehat{x}_{1}, \widehat{x}_{2}$, the former spatial coordinates:

$$
\left[\widehat{x}_{1}, \widehat{x}_{2}\right]=x_{1} \star x_{2}-x_{2} \star x_{1}=i \theta .
$$

The corresponding quantization of the area can be thought of as a discretization of the fluid (at the classical level), with the minimal area $\theta$ allocated to a single electron (cf.(26)).

\subsection{Matrix representation of the noncommutative Chern-Simons theory}

Every noncommutative theory is equivalent to a matrix theory, with matrices of infinite order $(N \rightarrow \infty)$ [24]; in particular, the noncommutative Chern-Simons theory (27) is equivalent to the matrix theory [25]:

$$
L=\frac{B}{2} \operatorname{Tr}\left[\varepsilon_{i j} X_{i}(t) D_{t} X_{j}(t)+2 \theta A_{0}(t)\right],
$$

where now $X_{1}(t), X_{2}(t)$ and $A_{0}(t)$ are $N \times N$ matrices $(N \rightarrow \infty)$ and the covariant derivative is $D_{t} X_{j}=\dot{X}_{j}-i\left[A_{0}, X_{j}\right], j=1,2$.

The proof of the correspondence is simpler if we go from the matrix (30) to the noncommutative theory (27). Observe that the Gauss law of the Lagrangian (30) implies the following noncommutative condition on the matrices:

$$
\left[X_{1}, X_{2}\right]=i \theta I,
$$

with $I$ the identity matrix. This algebra only admits $\infty$-dimensional matrix representations: consider a particular, "ground state" solution, $X=\hat{x}^{i}$, and write the general solutions as follows:

$$
X^{i}=\hat{x}^{i}+\theta \epsilon^{i j} A_{j}\left(\hat{x}^{i}\right),
$$

where $A_{i}$ are $N \times N$ matrices of "fluctuations" $(N \rightarrow \infty)$. Note that these matrices can be expressed in terms of finite sums of products $e^{i p \hat{x}^{1}} e^{i q \hat{x}^{2}}$, i.e. they can be thought 
of being functions of $\hat{x}^{i}$. Replacing (32) in (30) and expressing the derivative as commutators, $\left[\hat{x}^{i}, f\left(\hat{x}^{1}, \hat{x}^{2}\right)\right]=i \theta \epsilon^{i j} \partial_{j} f$, we find:

$$
\begin{aligned}
\mathcal{L}= & \frac{\mathbf{B}}{2} \operatorname{Tr}\left\{-\theta \dot{A}_{1}\left(\hat{x}_{1}+\theta A_{2}\right)+i A_{0}\left[\hat{x}_{1}+\theta A_{2}, \hat{x}_{2}-\theta A_{1}\right]\right. \\
& \left.\quad-\theta \dot{A}_{2}\left(\hat{x}_{2}-\theta A_{1}\right)-i A_{0}\left[\hat{x}_{2}-\theta A_{1}, \hat{x}_{1}+\theta A_{2}\right]\right\}+\mathbf{B} \theta A_{0} \\
= & \frac{\mathbf{B} \theta^{2}}{2} \operatorname{Tr}\left(A_{1} \dot{A}_{2}-\dot{A}_{1} A_{2}+2 A_{0}\left(\partial_{2} A_{1}-\partial_{1} A_{2}\right)+2 i A_{0}\left[A_{1}, A_{2}\right]\right),
\end{aligned}
$$

or in covariant notation,

$$
\mathcal{L}=\frac{\mathbf{B} \theta^{2}}{2} \operatorname{Tr}\left[-\epsilon^{\mu \nu \rho} A_{\mu} \partial_{\nu} A_{\rho}+\frac{2 i}{3} \epsilon^{\mu \nu \rho} A_{\mu} A_{\nu} A_{\rho}\right] .
$$

In the limit $N \rightarrow \infty$ the matrix variables $A_{i}$ are mapped into smooth functions of the noncommutative coordinates $A_{i}\left(\hat{x}_{j}\right)$. Also in this limit we can identify, $\theta \operatorname{Tr} \rightarrow$ $\frac{1}{2 \pi} \int d \hat{x}_{1} d \hat{x}_{2}$, and we obtain the following Lagrangian:

$$
\mathcal{L}=\frac{1}{4 \pi \nu} \int d \hat{x}_{1} d \hat{x}_{2}\left(-\epsilon^{\mu \nu \rho} A_{\mu} \partial_{\nu} A_{\rho}+\frac{2 i}{3} \epsilon^{\mu \nu \rho} A_{\mu} A_{\nu} A_{\rho}\right) .
$$

In this Lagrangian, the fields $A_{i}$ still obey the matrix algebra, while in (27) they are functions. The two formulations are related by expressing matrices as Wigner $c$-number functions that obey the Moyal product algebra [15].

Let us recall that any operator $\hat{F}\left(\hat{x_{1}}, \hat{x_{2}}\right)$ in the Weyl ordering : : (most symmetric in $\left.\hat{x_{1}}, \hat{x_{2}}\right)$ can be associated to a phase space function $F\left(x_{1}, x_{2}\right)$ as follows:

$$
\begin{aligned}
: \hat{F}\left(\hat{x_{1}}, \hat{x_{2}}\right): & =\int d x_{1} d x_{2} F\left(x_{1}, x_{2}\right): \delta\left(\hat{x_{1}}-x_{1}\right) \delta\left(\hat{x_{2}}-x_{2}\right): \\
& =\int d x_{1} d x_{2} \frac{d \alpha}{2 \pi} \frac{d \beta}{2 \pi} F\left(x_{1}, x_{2}\right) e^{i \alpha\left(\hat{x_{1}}-x_{1}\right)+i \beta\left(\hat{x_{2}}-x_{2}\right)} \\
& =\left.F\left(-i \frac{\partial}{\partial \alpha},-i \frac{\partial}{\partial \beta}\right) e^{i \alpha \hat{x_{1}}+i \beta \hat{x_{2}}}\right|_{\alpha=\beta=0} .
\end{aligned}
$$

One finds by inspection that the product of two operators $\hat{F}$ and $\hat{G}$ corresponds to the Moyal product (28) of the corresponding Wigner functions, : $\hat{F}:: \hat{G}:=: \hat{H}:$, $H\left(x_{1}, x_{2}\right)=(F \star G)\left(x_{1}, x_{2}\right)$. In particular,

$$
\int d \hat{x}_{1} d \hat{x}_{2}: \hat{F}\left(\hat{x}_{1}, \hat{x}_{2}\right):: \hat{G}\left(\hat{x}_{1}, \hat{x}_{2}\right):=\int d x_{1} d x_{2}(F \star G)\left(x_{1}, x_{2}\right) .
$$

Therefore, the matrix Lagrangian (35) becomes the noncommutative Chern-Simons theory (27) (within the Weyl ordering).

Finally, we recall that another route to obtain the Chern-Simons matrix theory (30), that emphasizes the discrete particle aspects of the fluid is given by a matrix regularization proposed by Goldstone and Hoppe [26].

\subsection{The Chern-Simons matrix model}

The noncommutative Chern-Simons Lagrangian (27) and its matrix model formulation (30) both imply infinite degrees of freedom: therefore, Susskind's theory applies to an 
infinite system. Instead, the fractional QHE is a system with a boundary and a finite number of particles. Polychronakos introduced this feature [9] by modifying Susskind's action (30) as follows:

$$
\begin{aligned}
S_{C S M M}= & \int d t \frac{\mathbf{B}}{2} \operatorname{Tr}\left\{\epsilon_{a b}\left(\dot{X}_{a}+i\left[A_{0}, X_{a}\right]\right) X_{b}+2 \theta A_{0}-\sigma X_{a}^{2}\right\} \\
& +\int d t \psi^{\dagger}\left(i \dot{\psi}-A_{0} \psi\right) .
\end{aligned}
$$

Two new terms are present: the first is a quadratic potential that confines the eigenvalues, i.e. keep the particles localized in the plane, with $\sigma=O(\mathbf{B} / N)$; the second term is a "boundary" $N$-dimensional complex vector $\psi$ that transforms in the fundamental representation of the gauge group $U(N)$. The Gauss law is now given by:

$$
G=-i \mathbf{B}\left[X_{1}, X_{2}\right]+\psi \psi^{\dagger}-\mathbf{B} \theta I=0 .
$$

Observe that the trace of (39) implies,

$$
\psi^{\dagger} \psi=N \mathbf{B} \theta,
$$

that can be realized with $N \times N$ dimensional matrices. The action (38) thus defines the Chern-Simons matrix model, a gauge theory with $U(N)$ symmetry, $X_{a} \rightarrow U X_{a} U^{\dagger}$,

$\psi \rightarrow U \psi$ and $A_{0} \rightarrow U A_{0} U^{\dagger}-i U \frac{d U^{\dagger}}{d t}$. Under a gauge transformation the action (38) is not invariant, but it yields a winding number, $S \rightarrow S-i \mathbf{B} \theta \int d t \operatorname{Tr}\left[U^{\dagger} \dot{U}\right]$; this requires the quantization $\mathbf{B} \theta=k$ [27], leading to the Laughlin filling fractions (cf.(26)). Note that the equation of motion for $\psi$ in the $A_{0}=0$ gauge read, $\dot{\psi}=0$, showing that this is an auxiliary field with trivial dynamics; it can take the constant value $\psi=\sqrt{N \mathbf{B} \theta}|v\rangle$, with $|v\rangle$ a vector of unit length [9].

\subsection{Covariant quantization}

In the $A_{0}=0$ gauge, the Hamiltonian of the Polychronakos theory (38) corresponds to $\left(N^{2}+N\right)$ particles in the lowest Landau level with coordinates $X_{n m}$ and $\psi_{n}$. It can be shown that, at quantum level, the Gauss law (39) implies gauge invariant states of the form $\Psi(X, \psi)=e^{-\operatorname{Tr}(\bar{X} X) / 2-\psi^{\dagger} \psi / 2} \Phi(X, \psi)$, with $\Phi(X, \psi)$ a singlet of the gauge group $U(N)$ made by polynomials of $X_{n m}$ and $\psi_{n}$, being of order $N k$ in $\psi_{n}$ due to (40) [10]. A basis of states is given by:

$$
\begin{aligned}
& \Phi(X, \psi)=\Phi_{\left\{n_{1}^{1}, \ldots, n_{N}^{1}\right\}} \ldots \Phi_{\left\{n_{1}^{k}, \ldots, n_{N}^{k}\right\}}, \\
& \Phi_{\left\{n_{1}^{j}, \ldots, n_{N}^{j}\right\}}=\epsilon^{i_{1} \ldots i_{N}}\left(X^{n_{1}^{j}} \psi\right)_{i_{1}} \ldots\left(X^{n_{N}^{j}} \psi\right)_{i_{N}}, \quad 0 \leq n_{1}^{j}<n_{2}^{j}<\ldots<n_{N}^{j} .
\end{aligned}
$$

These states are eigenstates of the angular momentum $J$ with eigenvalues $\mathcal{J}=N_{X}$, where $N_{X}$ is the number of matrices $X$ appearing in $\Phi(X, \psi)$. Since the Hamiltonian of the theory is proportional to the angular momentum, $H=(2 \omega / \mathbf{B}) J$, the states $(41)$ are also eigenstates of the Hamiltonian. The ground state of the theory is [10]:

$$
\Phi_{k-g s}=\left[\epsilon^{i_{1} \ldots i_{N}} \psi_{i_{1}}(X \psi)_{i_{2} \ldots}\left(X^{N-1} \psi\right)_{i_{N}}\right]^{k},
$$


corresponding to the lowest value of the angular momentum (lower order polynomials vanish by antisymmetry of the $\epsilon^{i_{1} \ldots i_{N}}$ tensor).

If we now diagonalize the matrix $X$ by the similarity transformation, $X=V^{-1} \Lambda V$, with $\Lambda=\operatorname{diag}\left(\lambda_{1}, \ldots, \lambda_{N}\right)$, we obtain:

$$
\Phi_{k-g s}\left(V^{-1} \Lambda V, \psi\right)=C \prod_{1 \leq n \leq m \leq N}\left(\lambda_{n}-\lambda_{m}\right)^{k} .
$$

The quantity $C$ appears in all the physical states and can be neglected in the present discussion [11].

Therefore we have obtained the Laughlin wave function as the ground state of the Chern-Simons theory, with electron coordinates identified with the eigenvalues of $X$. This is a very important result of the Chern-Simons matrix theory; that of reproducing the Laughlin wave function from gauge invariance of the states in presence of the "background charge" $\theta$. (Note that the filling fraction is $\nu=1 /(k+1)$ : the shift from the classical value (26) is due to a Vandermonde factor coming from the integration measure [9]).

Let us now discuss the excitations over the ground state (42). In Ref.[10], it was found the "bosonic" basis of states,

$$
\Phi(X, \psi)=\sum_{\left\{m_{k}\right\}} \operatorname{Tr}\left(X^{m_{1}}\right) \cdots \operatorname{Tr}\left(X^{m_{k}}\right) \Phi_{k-g s} .
$$

for any positive integers $\left\{m_{1}, \ldots, m_{k}\right\}$. These states have $\Delta \mathcal{J}=r=\sum_{k} m_{k}$. For $r=O(1)$, their energy given by the boundary potential, $\Delta E=\sigma \Delta \mathcal{J}=O(r \mathbf{B} / N)$ is very small: they are the edge excitations of the droplet of fluid described by conformal field theories [15][19][20].

The matrix model also possess localized density deformations that are analogues of the quasi-hole excitations of the Laughlin state. For example, the state $\Phi_{\left\{n_{1}, \ldots, n_{N}\right\}}$ in Eq.(41), with $\left\{n_{1}, n_{2}, \cdots, n_{M}\right\}=\{1,2, \cdots, N\}$, corresponds to moving one electron from the interior of the Fermi surface to the edge, causing $\Delta \mathcal{J}=O(N)$ and thus a finite gap $\Delta E=O(\mathbf{B})$. On the other hand, the quasi-particle excitation cannot be realized in the Chern-Simons matrix model [9][12][14], because excitations with angular momentum lower than (42) are zero due to the antisymmetry of the $\epsilon^{i_{1} \ldots i_{N}}$ tensor. Similarly, the Jain states $\nu=n /(2 n k+1)$ are not naturally obtained [28].

In conclusion, we have shown that the Chern-Simons matrix model reproduces the Laughlin wave functions as ground states. Nevertheless, the theory has some drawbacks [13]: there are no quasi-particle excitations [9], and the Jain states cannot be described [28]. Moreover, the measure of integration w.r.t. the eigenvalues $\lambda_{i}$ differs from that of electrons in the lowest Landau level, owing to the noncommutativity of matrices. It can be shown that the ground state properties of the matrix theory and of the Laughlin state only agree at long distances [11][29]. Owing to the inherent noncommutativity, it is also difficult to match matrix observables with electron quantities of the quantum Hall effect [30]. 


\subsection{Jain composite fermion transformation}

We would like to stress that the Chern-Simon matrix model provides another realization of the Jain composite-fermion transformation (see section 2.3). For $k=0$, the matrix theory reduced to the eigenvalues $\lambda_{a}$ is equivalent to a system of free fermions in the lowest Landau level, i.e. to $\nu^{*}=1$ [11][29]. This fermionic picture is a general feature of one-dimensional matrix models [31].

In the presence of the $\theta$ background, the noncommutativity of matrix coordinates (39) forces the electrons to acquire a finite area of order $\theta$, leading to the (semiclassical) density $\rho_{o}=1 / 2 \pi \theta$ (23). Using this formula of the density and the quantization of $\mathbf{B} \theta$, we re-obtain the Jain relation (13) for flux attachment,

$$
\mathbf{B} \theta=k \in \mathbb{Z} \rightarrow \mathbf{B}=k 2 \pi \rho_{o} .
$$

Given that noncommutativity is expressed by the Gauss law of the matrix theory, we understand that the mechanism for realizing the Jain transformation is analogous to that of the Lopez-Fradkin theory (cf. (18), section 2.4), but it is expressed in terms of different variables. However, the higher Landau levels are not possible in the ChernSimons matrix model.

\section{Maxwell-Chern-Simons matrix gauge theory}

In this section we introduce and analyze the Maxwell-Chern-Simons matrix theory [12] with the aim of improving the previous matrix theory. The action is,

$$
\begin{aligned}
S= & \int d t \operatorname{Tr}\left[\frac{m}{2}\left(D_{t} X_{i}\right)^{2}+\frac{\mathbf{B}}{2} \varepsilon_{i j} X_{i} D_{t} X_{j}+\frac{g}{2}\left[X_{1}, X_{2}\right]^{2}+\mathbf{B} \theta A_{0}\right] \\
& -i \int \psi^{\dagger} D_{t} \psi .
\end{aligned}
$$

It involves the same $N \times N$ Hermitean matrices, $X_{i}(t)$ and $A_{0}(t)$, and the auxiliary vector $\psi(t)$, but includes two new terms with respect to Polychronakos theory (38): an additional kinetic term quadratic in time derivatives and a potential $V=-g \operatorname{Tr}\left[X_{1}, X_{2}\right]^{2}$, parameterized by the positive coupling constant $g$. All the terms in the action are fixed by the gauge principle because they are obtained by dimensional reduction of a gauge theory. Indeed, the action (46) is the bosonic part of the low-energy effective theory of a stack of N D0-branes [32] that has been discussed in the literature of string theory [21]. In particular, D0-branes have been proposed as fundamental degrees of freedom in string theory [33].

\subsection{Low-energy effective action of Dp-branes in string theory}

Let us briefly review Witten's derivation of the effective low-energy action of $N$ Dpbranes [34]. Consider ten-dimensional Minkowski space, with time $x^{0}$ and space $x^{1}, \ldots, x^{9}$ coordinates, respectively. A p-brane is an object that modifies the boundary conditions 
of open strings: it introduces Dirichlet boundary conditions in $(9-p)$ directions, as follows,

$$
\begin{array}{ll}
X^{p+1}(\sigma, t)=\ldots=X^{9}(\sigma, t)=0 & \text { (Dirichlet) }, \\
\partial_{\sigma} X^{1}(\sigma, t)=\ldots=\partial_{\sigma} X^{p}(\sigma, t)=0 & \text { (Neumann). }
\end{array}
$$

Due to (47), the zero modes $X^{j}$ with $j>p$ are frozen, and the massless particles are functions of $X_{1}, \ldots, X_{p}$ only. The massless bosons $A_{i}\left(X^{s}\right), i, s=0, \ldots, p$, propagate as $U(1)$ gauge bosons on the p-brane, while the other components become scalars fields on the p-brane, $\phi_{j}\left(X^{s}\right), j>p$. The vertex operators for insertions of spin-1 fields in string theory are given by:

$$
\begin{aligned}
V_{A} & =\sum_{i=0}^{p} A_{i}\left(X^{s}\right) \partial_{\tau} X^{i}, \\
V_{\phi} & =\sum_{j>p} \phi_{j}\left(X^{s}\right) \partial_{\sigma} X^{j} .
\end{aligned}
$$

For $\phi_{j}=$ constant, the boundary integral of $V_{\phi}$ implies the change $X^{j} \rightarrow X^{j}+\phi^{j}$ for $j>p$ : thus the scalars $\phi_{j}, j>p$ can be interpreted as the coordinates of the p-brane. The theory on the $(p+1)$ dimensional brane world-volume is naturally the ten-dimensional $U(1)$ supersymmetric gauge theory dimensionally reduced to $(p+1)$ dimensions.

Bound states of $N$ parallel Dirichlet p-branes can be described by the low-energy limit when the branes are nearby. We consider the case of two parallel Dirichlet p-branes, one at $X^{j}=0$, and one at $X^{j}=a^{j}(j>p)$. The branes are connected by strings: they can start and end on the same brane and give a $U(1) \times U(1)$ gauge theory (with one $U(1)$ living one each p-brane), or they can start in the first brane and end in the second (and viceversa). In this case, the strings have $U(1) \times U(1)$ charges. The ground state of this configuration has an energy $T|a|$, with $T$ and $|a|$ being the tension and length of the string, respectively. When $|a| \rightarrow 0$ the charged vector bosons become massless and the $U(1) \times U(1)$ gauge symmetry is enlarged to a $U(2)$ symmetry. In the same way, $N$ coincident parallel branes yield a $U(N)$ gauge symmetry on the p-brane. The field content in the effective action is given by the $U(N)$ gauge field $A_{j}\left(X^{s}, t\right)$, with $s, j=1, \ldots, p$, and the scalar fields $\phi_{j}\left(X^{s}, t\right)$, with $j>p$, in the adjoint representation of $U(N)$, i.e. they are all $N \times N$ matrices.

The reduction to $(p+1)$ dimensions of the bosonic sector of the theory is obtained as follows. From the Lagrangian,

$$
L_{Y M}=-\frac{1}{4 g^{2}} \operatorname{Tr}\left(F^{\mu \nu} F_{\mu \nu}\right)
$$

we simplify the commutators in $F^{\mu \nu}=\left[D_{\mu}, D_{\nu}\right]$ by dimensional reduction and identify the earlier fields, leading to:

$$
L_{Y M}^{\prime}=-\frac{1}{4 g^{2}} \operatorname{Tr}\left(\sum_{r, s=0}^{p} F^{r s} F_{r s}-\sum_{s=1}^{p} \sum_{j>p} D_{s} \phi_{j} D^{s} \phi^{j}+\sum_{i, j>p}\left[\phi_{i}, \phi_{j}\right]^{2}\right) .
$$

In the $p=0$ case we have D0-branes, that are nonrelativistic point particle with matrix variables and one-dimensional gauge symmetry. In (2+1)-dimensions, (51) becomes 
the Maxwell-Chern-Simons theory (46), apart from the Chern-Simons kinetic term: as shown in Ref.[21], this can be obtained by adding a configuration of higher D-branes that creates a magnetic field for the D0-branes.

\subsection{Covariant quantization of Maxwell-Chern-Simons theory}

In this section we quantize the Maxwell-Chern-Simons matrix theory (46) [12][35]. The canonical momenta are given by the Hermitean matrices:

$$
\Pi_{i}=\frac{\delta S}{\delta \dot{X}_{i}^{T}}=D_{t} X_{i}-\frac{\mathbf{B}}{2} \varepsilon_{i j} X_{j}
$$

and the vector $\chi=\delta S / \delta \dot{\psi}=-i \psi^{\dagger}$. The Hamiltonian is:

$$
H=\operatorname{Tr}\left[\frac{1}{2}\left(\Pi_{i}+\frac{\mathbf{B}}{2} \varepsilon_{i j} X_{j}\right)^{2}-\frac{g}{2}\left[X_{1}, X_{2}\right]^{2}\right] .
$$

The Gauss law constraint now reads:

$$
G=0, \quad G=i\left[X_{1}, \Pi_{1}\right]+i\left[X_{2}, \Pi_{2}\right]-\mathbf{B} \theta I+\psi \otimes \psi^{\dagger} .
$$

As in Chern-Simons theory, G generates $U(N)$ gauge transformations on $X_{i}$ and $\psi$ at the quantum level, and requires the physical states to be $U(N)$ singlets. We now quantize all the $2 N^{2}$ matrix degrees of freedom $X_{a b}^{i}$ and later impose the Gauss law as a differential condition on wave functions. It is useful to introduce holomorphic coordinates:

$$
\begin{array}{llrl}
X & =X_{1}+i X_{2}, & \bar{X} & =X_{1}-i X_{2}, \\
\Pi & =\frac{1}{2}\left(\Pi_{1}-i \Pi_{2}\right), & \bar{\Pi}=\frac{1}{2}\left(\Pi_{1}+i \Pi_{2}\right),
\end{array}
$$

with the bar denoting the Hermitean conjugate of classical matrices, keeping the dagger for the quantum adjoint.

The Hamiltonian (53) for $g=0$ is quadratic and easily solvable: introduce the matrix,

$$
A_{a b}=\frac{1}{2 \ell} X_{a b}+i \ell \bar{\Pi}_{a b}
$$

and its adjoint $A^{\dagger}$. Owing to the canonical commutators, they obey the algebra of $N^{2}$ harmonic oscillators:

$$
\left[\left[A_{a b}, A_{c d}^{\dagger}\right]\right]=\delta_{a d} \delta_{b c}, \quad\left[\left[A_{a b}, A_{c d}\right]\right]=0 .
$$

In this following, the double brackets describe quantum commutators while the single ones are kept for the matrix algebra; note also that $A^{\dagger}$ is the adjoint of $A$ both as a matrix and a quantum operator. The Hamiltonian can be expressed in term of $A$ and $A^{\dagger}$ as follows:

$$
H=\mathbf{B} \operatorname{Tr}\left(A^{\dagger} A\right)+\frac{\mathbf{B}}{2} N^{2}+\frac{g}{8} \operatorname{Tr}[\bar{X}, X]^{2} .
$$

In the term $\operatorname{Tr}\left(A^{\dagger} A\right)=\sum_{a b} A_{a b}^{\dagger} A_{b a}$ one recognizes $N^{2}$ copies of the Landau level Hamiltonian corresponding to $N^{2}$ two-dimensional "particles" with phase-space 
coordinates, $\left\{\Pi_{a b}, X_{a b}\right\}$ and $\left\{\bar{\Pi}_{a b}, \bar{X}_{a b}\right\}, a, b=1, \ldots, N$. The one-particle state are similarly characterized by another set of independent oscillators corresponding to angular momentum excitations, that are described by the matrix $B=\bar{X} / 2 \ell+i \ell \Pi$ and its adjoint $B^{\dagger}$, obeying the algebra:

$$
\left[\left[B_{a b}, B_{c d}^{\dagger}\right]\right]=\delta_{a d} \delta_{b c}, \quad\left[\left[B_{a b}, B_{c d}\right]\right]=0 .
$$

The total angular momentum of the $N^{2}$ "particles" can be written in the $U(N)$ invariant form,

$$
J=\frac{1}{i} \operatorname{Tr}(\bar{X} \bar{\Pi}-X \Pi)=\operatorname{Tr}\left(B^{\dagger} B-A^{\dagger} A\right) .
$$

For large values of the magnetic field $\mathbf{B}$, the reduction of the theory (46) to the lowest Landau is obtained by imposing $A_{a b} \approx 0, \forall a, b$ : the theory becomes the previously studied Chern-Simons matrix model (38), because the quadratic kinetic term vanishes and the potential reduces to a constant due to the Gauss law.

\section{Matrix ground states at $g=0$}

\subsection{Jain states by projections}

The gauge invariant states can be written,

$$
\Psi=e^{-\operatorname{Tr}(\bar{X} X) / 2-\bar{\psi} \psi / 2} \Phi(X, \bar{X}, \psi),
$$

where $\Phi(X, \bar{X}, \psi)$ is again a $U(N)$ singlet made of matrices $X, \bar{X}$ and $N k$ components of the vector $\psi$. The general solutions (61) are similar to those obtained in Chern-Simons theory with the difference that now the polynomial part also depends on the $\bar{X}$ matrices; for example,

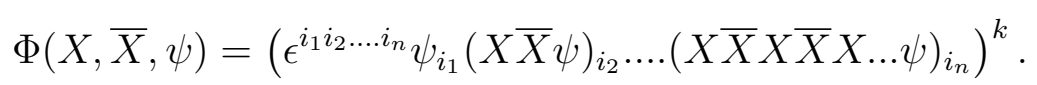

It is better to express these polynomials in terms of the variables $\bar{A}$ and $\bar{B}$ (cf. section 4.2): from the commutation relations $(57,59)$, the energy and momentum eigenstates can

be easily obtained by applying the $A_{a b}^{\dagger}(56)$ and $B_{a b}^{\dagger}$ operators to the empty ground state $\Psi_{o}=\exp (-\operatorname{Tr} \bar{X} X / 2-\bar{\psi} \psi / 2)$. Their energy $E=\mathbf{B} N_{A}$ and momentum $\mathcal{J}=N_{B}-N_{A}$ are expressed in terms of the number of $A^{\dagger}$ and $B^{\dagger}$ operators, $N_{A}$ and $N_{B}$ respectively. The wave functions is rewritten:

$$
\Psi=e^{-\operatorname{Tr} \bar{X} X / 2-\bar{\psi} \psi / 2} \Phi(\bar{B}, \bar{A}, \psi), \quad E=\mathbf{B} N_{A}, \quad J=N_{B}-N_{A},
$$

where $\bar{B}=X-\partial / \partial \bar{X}$ and $\bar{A}=\bar{X}-\partial / \partial X$ commute among themselves, $\left[\left[\bar{A}_{a b}, \bar{B}_{c d}\right]\right]=0$, and can be treated as $c$-number matrices. Any polynomial $\Phi(\bar{B}, \bar{A}, \psi)$ yields an energy eigenstate and corresponds in general to a sum of terms (62). Let us remark that for states with constant density, the angular momentum measures the extension of the "droplet of fluid", such that we can associate a corresponding filling fraction $\nu$ by the formula,

$$
\nu=\lim _{N \rightarrow \infty} \frac{N(N-1)}{2 \mathcal{J}} .
$$




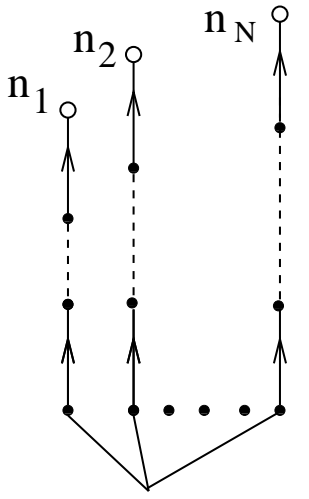

( a )

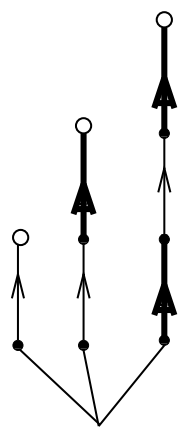

( b )

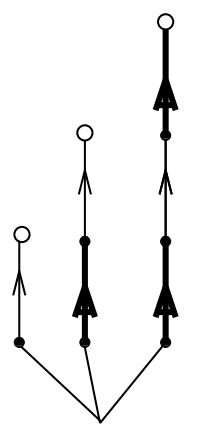

( c )

Figure 4. Graphical representation of gauge invariant states: (a) general states in the lowest Landau level (cf. Eq.(41)); (b) and (c), $N=3$ examples involving both matrices, $\bar{B}$ (thin line) and $\bar{A}$ (in bold).

The states (63) can be represented graphically as "bushes", as shown in Fig.4(a). The matrices $\bar{B}_{a b}$ (i.e. $X_{a b}$ ) are depicted as oriented segments with indices at their ends and index summation amounts to joining segments into lines, as customary in gauge theories. The matrices $\bar{A}_{a b}$ are represented by bold segments. The lines are the "stems" of the bush ending with one $\psi_{a}$, represented by an open dot, and the epsilon tensor is the N-vertex located at the root of the bush. Bushes have N stems of different lengths: $n_{1}<n_{2}<\cdots<n_{N}$. The position $i_{\ell}$ of one $\bar{B}$ on the $\ell$-th stem, $1 \leq i_{\ell} \leq n_{\ell}$, is called the "height" on the stem. Since two stems cannot be equal, one obtains a kind of Fermi sea of $N$ "one-particle states" corresponding to the $N$ strands. However, there are additional degeneracies with respect to an ordinary fermionic system, because in each stem all possible words of $\bar{A}$ and $\bar{B}$ of given length yield independent states (for large $N$ ), owing to matrix noncommutativity, as seen in fig.4(b) and 4(c).

The complete filling of all the available degenerate $E>0$ states at $g=0$ clearly gives very dense and inhomogeneous fluids that are incompatible with the physics of the quantum Hall effect. The matrix degeneracies lead to a density of states that grows exponentially with the energy; this is a characteristic of string theories that is not suitable for the Hall effect [33]. On the other hand, for $g>0$ the potential $\operatorname{Tr}[X, \bar{X}]^{2}$ in the Hamiltonian (58) constraints matrix noncommutativity and eventually eliminates the degeneracy: at $g=\infty$, this is not present and the theory can describe a physical electron system, as shown in section 6 .

Given that the $g>0$ theory is difficult to solve, in ref. [12] we introduced a set of projections that limit the matrix degeneracy at $g=0$ and are explicitly solvable. These projections are expressed by the following constraints on the wave function,

$$
\left(A_{a b}\right)^{m} \Psi=0 \longrightarrow\left(\frac{\partial}{\partial \bar{A}_{a b}}\right)^{m} \Phi(\bar{A}, \bar{B}, \psi)=0, \quad \forall a, b
$$


for a given value of $m$. The $m=1$ case is the lowest Landau level discussed before with no $\bar{A}$ dependence, while $m$ taking successive values $m=2,3, \ldots$ gradually allow larger $\bar{A}$ multiplicities and thus matrix degeneracies. Note that in equation (65), each matrix component $A_{a b}$ is raised to the $m$-th power, without index summation: the condition is nevertheless gauge invariant and admits an equivalent manifestly invariant form that is discussed later.

The results of ref.[12] were rather interesting: not only the projections (65) allow homogeneous ground states suitable for describing quantum Hall fluids, but also they precisely occur in the Jain pattern of filling fractions, $\nu=m /(m k+1)$, and their derivation repeats step-by step the Jain "composite fermion" construction [5].

Let us recall the main points of the analysis. Consider first the projection (65) for $m=2$ and choose $k=1$ : the solutions are polynomials that are at most linear in each component $\bar{A}_{a b}$. Let us imagine that one or more $\bar{A}$ matrices are present at points on the bush as in Fig.(4). The differential operator (65) acts by sequentially erasing pairs of bold lines on the bush, each time detaching two strands and leaving four free extrema with indices fixed to either $a$ or $b$, with no summation on them. For example, when acting on a pair of $\bar{A}$ located on the same stem, it yields a non-vanishing result: this limits the bushes to have one $\bar{A}$ per stem at most. The $A^{2} \approx 0$ conditions can be satisfied if cancellations occur for pairs of $\bar{A}$ on different stems, as it follows:

$$
\left(A_{a}^{b}\right)^{2} \Phi=\cdots+\varepsilon^{\cdots i \ldots j \cdots}\left(\cdots M_{i a} N_{j a} \cdots V^{b} W^{b}\right)+\cdots, \quad(a, b \text { fixed }) .(66)
$$

This expression vanishes for $M=N$ due to the antisymmetry of the epsilon tensor. The general solution of (65) is given by bushes involving one $\bar{A}$ per stem at most (max $\mathrm{N}$ matrices in total), with all of them located at the same height on the stems [12]. In formulas:

$$
\begin{gathered}
\Phi_{\left\{n_{1}, \ldots, n_{\ell} ; p ; n_{\ell+1}, \ldots, n_{M}\right\}}=\varepsilon^{i_{1} \ldots i_{N}} \prod_{k=1}^{\ell}\left(\bar{B}^{n_{k}} \psi\right)_{i_{k}} \prod_{k=\ell+1}^{N}\left(\bar{B}^{p} \bar{A} \bar{B}^{n_{k}} \psi\right)_{i_{k}}, \\
0 \leq n_{1}<\cdots<n_{\ell}, \quad 0 \leq n_{\ell+1}<\cdots<n_{N} .
\end{gathered}
$$

If the matrices $\bar{A}, \bar{B}$ were diagonal, these states would be Slater determinants of ordinary Landau levels. The matrix states have further degeneracies by commuting $\bar{A}, \bar{B}$ pairs: however, commutations are severely limited in the the solution (67), only the global $p$ dependence is allowed. This shows how the $A^{2} \approx 0$ projection works in reducing matrix degeneracies.

The ground state in the $A^{2} \approx 0$ theory with finite-box boundary conditions is the lowest $J$ states of the form (67): it corresponds to homogeneous filling all the allowed states in the first and second Landau levels with $N / 2$ "gauge invariant particles" each, and reads:

$$
\Phi_{1 / 2, g s}=\varepsilon^{i_{1} \ldots i_{N}} \prod_{k=1}^{N / 2}\left(\bar{B}^{k-1} \psi\right)_{i_{k}} \prod_{k=1}^{N / 2}\left(\bar{A} \bar{B}^{k-1} \psi\right)_{i_{N / 2+k}},
$$

with angular momentum $\mathcal{J}=N(N-4) / 4$. This state is non-degenerate due to the vanishing of the $p$ parameter in (67). It has filling fraction $\nu^{*}=2$, assuming homogeneity 
of its density, to be shown later.

The ground states for $k>1$ are products of $k$ bushes: they obey the constraint $A^{2} \approx 0$ provided that the two derivatives always vanish when distributed over the bushes. Given one bush of type (68), obeying $A^{2} \Phi_{1 / 2, g s}=0$, one can form the state,

$$
\Phi_{k+1 / 2, g s}=\Phi_{k-1, g s} \Phi_{1 / 2, g s},
$$

where the other $(k-1)$ bushes satisfy $A \Phi_{k-1, g s}=0$ and actually are Laughlin's one (41). Using (64), the angular momentum of this state corresponds to the filling fraction $1 / \nu=k+1 / 2$.

We thus find the important result that the $A^{2} \approx 0$ projected Maxwell-Chern-Simons theory possesses non-degenerate ground states that are the matrix analogues of the Jain states obtained by composite-fermion transformation at $\nu^{*}=2$, i.e. $1 / \nu=1 / \nu^{*}+k$. The matrix states $(69,68)$ would actually be exactly equal to Jain's wave functions, if the $\bar{A}, \bar{B}$ matrices were diagonal: the $\psi$ dependence would factorize and the matrix states reduce to the Slater determinants of the Jain wave functions [5][16] (cf. (14) in section $2.3)$.

The correspondence extends to the whole Jain series: the other $\nu^{*}=m$ nondegenerate ground states are respectively obtained in the theories with $A^{m} \approx 0$ projections. They read:

$$
\Phi_{k+1 / m, g s}=\Phi_{k-1, g s} \Phi_{1 / m, g s}
$$

where,

$$
\Phi_{1 / m, g s}=\varepsilon^{i_{1} \ldots i_{N}} \prod_{k=1}^{N / m}\left[\left(\bar{B}^{k-1} \psi\right)_{i_{k}}\left(\bar{A} \bar{B}^{k-1} \psi\right)_{i_{k+N / m}} \cdots\left(\bar{A}^{m-1} \bar{B}^{k-1} \psi\right)_{i_{k+(m-1) N / m}}\right] .(71
$$

In conclusion, in ref.[12] we found that the ground states of the properly projected Maxwell-Chern-Simons matrix theory reproduce the Jain pattern of the composite fermion construction [5]; the matrix states are non-degenerate for specific values of the density that are controlled by the boundary potential [12]. These results indicate that the Jain composite fermions have some relations with the D0-brane degrees of freedom and their underlying gauge invariance. Both of them have been described as dipoles: according to Jain [5] and Haldane-Pasquier [36], the composite fermion can be considered as the bound state of an electron and a hole (a vortex in the electron fluid). On the other side, matrix gauge theories, and the equivalent noncommutative theories [33], describe D0 branes that are point-like dipoles in the low-energy limit of string theory.

A final remark on the noncommutative matrix coordinates in the Jain and Laughlin state: the Gauss law (54) can be rewritten in terms of $X, \bar{X}, A, \bar{A}$ as follows:

$$
[X, \bar{X}]+\frac{2}{B}[\bar{X}, A]+\frac{2}{B}[\bar{A}, X]=2\left(\theta-\frac{1}{B} \psi \otimes \bar{\psi}\right) .
$$

For the Laughlin states in the lowest Landau level, this reduces to coordinates noncommutativity (39), because $A=\bar{A}=0$; for the Jain states populating higher levels, 
there are other terms contributing to noncommutativity besides the matrix coordinates, such that higher density values are possible.

\subsection{Gauge invariant form of the projections}

Although the operators $\left(A_{a b}\right)^{m}, m=1,2, .$. , are not explicitly gauge invariant, their kernel restricted to gauge invariant states yields gauge invariant conditions, as seen in the previous discussion. Therefore, the projectors should have manifestly gauge invariant expressions. In Ref. [14], they were found by expressing the conditions $A^{m} \approx 0$ in terms of positive-definite occupation numbers $Z_{a b}=A_{a b}^{\dagger} A_{a b}$ (no sum over $a, b$ ), and by averaging over their gauge orbit. For $m=2$, the $A^{2} \approx 0$ constraint was shown to be equivalent to $Q_{2}^{g . i .} \approx 0$, with:

$$
Q_{2}^{g . i .} \propto\left(\delta_{k i} \delta_{l j}+\delta_{k j} \delta_{l i}\right) A_{i a^{\prime}}^{\dagger} A_{j a}^{\dagger} A_{a k} A_{a^{\prime} l} .
$$

Upon commuting the operators to bring summed indices close to each other, we finally find the manifestly gauge-invariant form (disregarding the normalization):

$$
Q_{2}^{g . i .}=\operatorname{Tr}\left(A^{\dagger} A A^{\dagger} A\right)+\left(\operatorname{Tr} A^{\dagger} A\right)^{2}-(N+1) \operatorname{Tr}\left(A^{\dagger} A\right) .
$$

One can check that the action of the gauge-invariant constraint $Q_{2}^{g . i .}$ on bush wave functions is completely equivalent to that of the gauge-variant condition $A^{2} \approx 0[12]$. The expressions (73) easily generalizes to higher $m$ values [14].

\subsection{Semiclassical solutions at $g=0$}

In this section we review the semiclassical analysis of the $g=0$ Maxwell-ChernSimons theory: in Ref. [14], we found the semiclassical states that correspond to the quantum states with homogeneous filling and composite-fermion structure (70) of the previous section and some of their quasiparticle excitations. The motivations for the semiclassical analysis are twofold: on one side, previous experience [9][13][30] [33][37] with noncommutative field theory has shown that the classical fluid dynamics incorporates some properties of the full quantum theory. From another side, it is know that the Laughlin states in the quantum Hall effect are incompressible fluids that become semiclassical in the thermodynamic limit $N \rightarrow \infty[15]$.

As we showed in section 5.1, the Jain-like ground states (70) involve higher Landau levels $(A \neq 0)$ and have filling fractions $\nu^{*}=2,3, \ldots$ (cf. (13) in section 2.3). We first note that these states are characterized by $E=O(N)$ and $J=O\left(N^{2}\right)$, thus implying that the matrix $A$ must have few nonvanishing elements $O(1)$. Indeed, the constraint $A^{m} \approx 0$ can be written in terms of occupation numbers, $Z_{a b}=\bar{A}_{a b} A_{a b}$, and limit the semiclassical values of $A_{a b}$ matrix elements: once summed over each row or column, they can take the values $\gamma=0,1, \ldots, m-1[14]$.

We introduce the constraints of the Gauss law and the projection $A^{m} \approx 0$ in the Hamiltonian with Lagrange multipliers $\Lambda$ and $\Gamma_{a}, \Gamma_{b}^{\prime}$, respectively. Upon variation with 
respect to $\bar{A}, \bar{B}$, we obtain the equations of motion:

$$
\begin{array}{rlrl}
i \dot{A}_{a b} & =2 A_{a b}-[\Lambda, A]_{a b}+A_{a b}\left(\Gamma_{a}+\Gamma_{b}^{\prime}\right), & \\
i \dot{B} & =-[\Lambda, B]+\omega B, & \\
G & =[\bar{A}, A]+[\bar{B}, B]-k+\psi \otimes \bar{\psi}=0, \\
Z_{a} & =\sum_{b} \bar{A}_{b a} A_{a b}=\gamma, & \gamma=0,1, \ldots, m-1, \\
Z_{b}^{\prime} & =\sum_{a} \bar{A}_{b a} A_{a b}=\gamma^{\prime}, & \gamma^{\prime}=0,1, \ldots, m-1 .
\end{array}
$$

The semiclassical ground states correspond to solutions with $\dot{A}=\dot{B}=0$.

Let us first recall the classical ground state with $A_{a b} \approx 0$ (lowest Landau level) found by Polychronakos in the Chern-Simons matrix model [9]: in this case $(\ell=1)$, $\bar{B}=X$ and $\psi$ are given by,

$$
\bar{X}=\sqrt{k} \sum_{n=1}^{N-1} \sqrt{n}|n\rangle\langle n-1|, \quad \psi=\sqrt{k N}| N-1\rangle .
$$

(denoting $|0\rangle, \cdots,|N-1\rangle$ the basis vectors [14]). The radius-squared matrix coordinate $R^{2}$ is diagonal, and given by:

$$
R^{2}=\bar{X} X=\operatorname{diag}(0, k, 2 k, \ldots,(N-1) k) .
$$

From the distribution of the eigenvalues in (77), it is clear that this solution implies an uniform density. In the large $\mathrm{N}$ limit, the filling fraction takes the Laughlin values $\nu=1 / k$ according to the formula (64).

In general, the one-particle density of rotation invariant states in matrix models can be defined in terms of the gauge invariant eigenvalues of $R^{2}$, as follows $\left(\rho(r)=\rho\left(r^{2}\right) / \pi\right)$ :

$$
\rho\left(r^{2}\right)=\sum_{i=0}^{N-1} \delta\left(r^{2}-\sigma_{i}\right), \quad \sigma_{i} \in \operatorname{Spec}\left(R^{2}\right) .
$$

For semiclassical fluids, this becomes a piecewise continuous function in the limit $N \rightarrow \infty$. A discrete approximation suitable for the continuum limit is [14]:

$$
\rho\left(r^{2}\right)=\sum_{i} \frac{n_{i}}{\sigma_{i+1}-\sigma_{i}} \delta_{r^{2}, \sigma_{i}},
$$

involving the Kronecker delta and the ordered set of distinct eigenvalues, $\sigma_{i}<\sigma_{j}, i<j$, with multiplicities $n_{i}$.

From Ref.[9], we also recall the form of the quasi-hole in the origin, in the lowest Landau level:

$$
\bar{X}=\sqrt{k}\left(\sqrt{q}|0\rangle\left\langle N-1\left|+\sum_{n=1}^{N-1} \sqrt{n+q}\right| n\right\rangle\langle n-1|\right), \quad q>0,
$$

where $q$ is proportional to the charge of the quasi-hole. The $R^{2}$ eigenvalues are correspondingly shifted upward by $q$, causing a dip at the origin.

The semiclassical ground state solution for $A^{2} \approx 0$, leading to the Jain like $\nu^{*}=2$ ground state is found by a suitable ansatz to the equation of motions (75). After gauge 
(a)

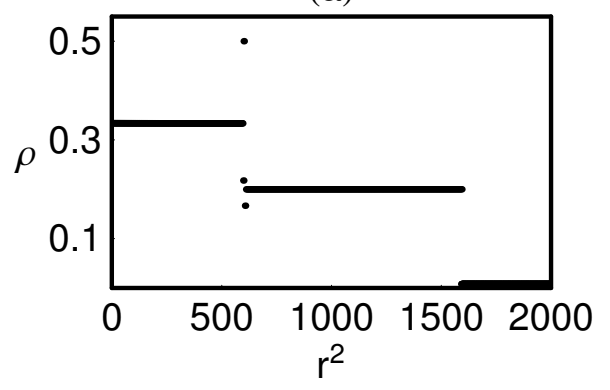

(b)

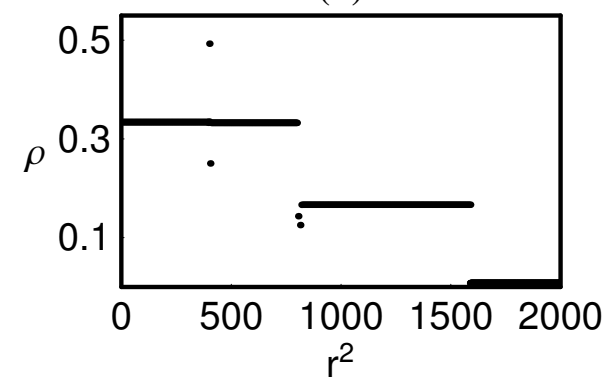

(c)

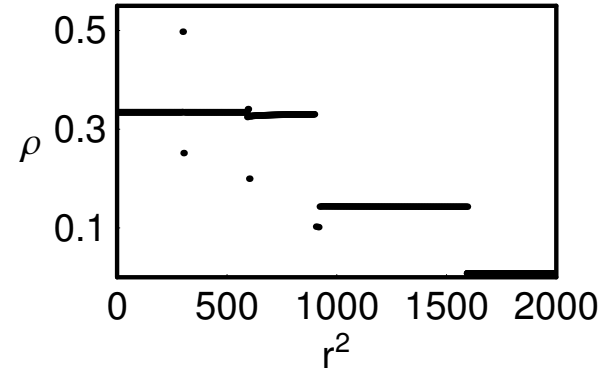

Figure 5. Density plots for the matrix ground states with $1 / \nu_{c l}=1 / \nu^{*}+k$, for $k=4$ and $N=400$ : (a) $\nu^{*}=2(81)$; (b) $\nu^{*}=3$; and (c) $\nu^{*}=4$.

choice, they imply that: i) $B$ is again a raising operator as in (76) and ii) $A$ has only one element 1 in each row and column, i.e. it is a (partial) permutation matrix. Using these data, the matrix equations can be reduced to a linear system of $O(N / 2)$ conditions leading to the solution ( $N$ even):

$$
\begin{aligned}
& B=\sum_{n=1}^{N / 2} \sqrt{n(k-1)}|n\rangle\left\langle n-1\left|+\sum_{n=\frac{N}{2}+1}^{N-1} \sqrt{n(k+1)-N}\right| n\right\rangle\langle n-1| \\
& A=\sum_{n=0}^{\frac{N}{2}-1}\left|n+\frac{N}{2}\right\rangle\langle n|
\end{aligned}
$$

with $\psi$ as in (76). In matrix form for $N=4$, it reads:

$$
B=\left(\begin{array}{cccc}
0 & 0 & 0 & 0 \\
\sqrt{k-1} & 0 & 0 & 0 \\
0 & \sqrt{2(k-1)} & 0 & 0 \\
0 & 0 & \sqrt{3 k-1} & 0
\end{array}\right), \quad A=\left(\begin{array}{cccc}
0 & 0 & 0 & 0 \\
0 & 0 & 0 & 0 \\
1 & 0 & 0 & 0 \\
0 & 1 & 0 & 0
\end{array}\right) .
$$

This solution has same energy $E=\mathbf{B} N / 2$ of the quantum state (69) and same angular momentum $J=(k-1 / 2) N^{2} / 2+O(N)$ to leading order. The matrix $R^{2}=(B+\bar{A})(\bar{B}+A)$ contains off-diagonal terms from the mixed products: however, these give subdominant $O(1 / \sqrt{N})$ corrections to the eigenvalues as is clear in a simple two-by-two matrix example. Thus, $\operatorname{Spec}\left(R^{2}\right)=\operatorname{Spec}(B \bar{B})(1+O(1 / \sqrt{N}))$.

In Fig. 5 we plot the densities of the $\nu^{*}=2$ ground state (81), and those of the corresponding $\nu^{*}=3$, 4 states [14], for $N=400$ : up to finite- $N$ fluctuations, they show 
two-step uniform densities as anticipated. We recall that the same droplet shape is found for the Jain states (14), before their projection to the lowest Landau level [5].

In Ref.[14] we found a simple argument for the equivalence of the semiclassical solutions to the matrix wavefunction found in section 5 (cf. (67)). We evaluated their

polynomial parts $\Phi(\bar{A}, \bar{B}, \psi)$ on the classical solution $\bar{A}, \bar{B}$, e.g. (81), corresponding to the leading $N \rightarrow \infty$ expectation values. We then found that the resulting polynomial selfconsistently match the single particle occupancies predicted by the classical solution themselves. These results confirm the validity of the semiclassical approximation for these matrix ground states.

\section{Electrons from D0 branes in the $g \rightarrow \infty$ limit}

\subsection{The matrix theory at $g=\infty$}

In this section we introduce the potential $V=-(g / 2) \operatorname{Tr}\left[X_{1}, X_{2}\right]^{2}$ in the Hamiltonian (53) and perform the $g \rightarrow \infty$ limit. At the classical level, the $V$ potential suppresses the matrix degrees of freedom different from the eigenvalues, and projects them out for $g \rightarrow \infty$. Using the Ginibre decomposition of complex matrices [31]: $X=\bar{U}(\Lambda+R) U$, where $U$ is unitary (the gauge degrees of freedom), $\Lambda$ diagonal (the eigenvalues) and $R$ complex upper triangular (the additional d.o.f.), we find for $N=2$ :

$$
V=\frac{g}{8} \operatorname{Tr}[X, \bar{X}]^{2}=\frac{g}{4}|r|^{4}+\frac{g}{4}\left|\bar{r}\left(\lambda_{1}-\lambda_{2}\right)\right|^{2}, \quad X=\left(\begin{array}{cc}
\lambda_{1} & r \\
0 & \lambda_{2}
\end{array}\right) .
$$

Thus for large $g$, the variable $r$ is suppressed. For general $N$, the potential kills all the $N(N-1)$ degrees of freedom contained in the $R$ matrix.

Let us now discuss the Maxwell-Chern-Simons theory in the $g=\infty$ limit, i.e. for $R=0: \quad X$ and $\bar{X}$ commute among themselves and can be diagonalized by the same unitary transformation,

$$
X=\bar{U} \Lambda U, \quad \bar{X}=\overline{U \Lambda} U, \quad \Lambda=\operatorname{diag}\left(\lambda_{a}\right), \quad[X, \bar{X}]=0 .
$$

The $g=\infty$ theory is analyzed following a different strategy from that of section 4 : we fix gauge invariance, solve the Gauss law at the classical level and then quantize the remaining variables, which are the complex eigenvalues $\lambda_{a}$ and their conjugate momenta $p_{a}$ [38][35]. We take the diagonal gauge for the coordinates and decompose the momenta $\Pi, \bar{\Pi}$, in diagonal and off-diagonal matrices, respectively called $p$ and $\Gamma$ : $X=\Lambda, \Pi=p+\Gamma, \bar{\Pi}=\bar{p}+\bar{\Gamma}$. The Gauss law constraint (54) can be rewritten:

$$
\begin{aligned}
{[X, \Pi]+[\bar{X}, \bar{\Pi}] } & =-i \mathbf{B} \theta+i \psi \otimes \bar{\psi}, \\
\left(\lambda_{a}-\lambda_{b}\right) \Gamma_{a b}+\left(\bar{\lambda}_{a}-\bar{\lambda}_{b}\right) \bar{\Gamma}_{a b} & =-i\left(k \delta_{a b}-\psi_{a} \bar{\psi}_{b}\right) .
\end{aligned}
$$

For $a=b$, this equation implies $\left|\psi_{a}\right|^{2}=k$ for any value of $a$ : for $a \neq b$, it completely determines the off-diagonal momenta:

$$
\Gamma_{a b}=\frac{i k}{2} \frac{\bar{\lambda}_{a}-\bar{\lambda}_{b}}{\left|\lambda_{a}-\lambda_{b}\right|^{2}}, \quad a \neq b .
$$


By inserting this back into the Hamiltonian (53), we find that diagonal and off-diagonal terms decouple and obtain,

$$
\begin{aligned}
H_{g=\infty} & =2 \operatorname{Tr}\left[\left(\frac{\bar{X}}{2}-i \Pi\right)\left(\frac{X}{2}+i \bar{\Pi}\right)\right] \\
& =2 \sum_{a=1}^{N}\left(\frac{\bar{\lambda}_{a}}{2}-i p_{a}\right)\left(\frac{\lambda_{a}}{2}+i \bar{p}_{a}\right)+\frac{k^{2}}{2} \sum_{a \neq b=1}^{N} \frac{1}{\left|\lambda_{a}-\lambda_{b}\right|^{2}} .
\end{aligned}
$$

Therefore, the theory reduced to the eigenvalues corresponds to the ordinary Landau problem for $\mathrm{N}$ electrons plus an induced two-dimensional Calogero interaction. Note also that the matrix measure of integration becomes flat after incorporating one Vandermonde factor $\Delta(\lambda)$ into the wave functions. The occurrence of the Calogero interaction is a rather common feature of matrix theories reduced to eigenvalues: in the present case, the interaction is two-dimensional, owing to the presence of two Hermitean matrices, and thus it is rather different from the exactly solvable one-dimensional case [9][39].

We conclude that the Maxwell-Chern-Simons matrix theory at $g=\infty$ makes contact with the physical problem of the fractional quantum Hall effect. The $e^{2} / r$ Coulomb repulsion is replaced by the Calogero interaction $k^{2} / r^{2}$; however, numerical results [3][5][16] indicate that quantum Hall incompressible states are rather independent of the type of repulsive potential, for large B. (The specific form of the potential clearly affects the detailed values of some quantities such as the gap.)

Some remarks are in order:

- The physical condition imposed by the Gauss law (85) is still that outlined in section 3.2.1: it forces the electrons to stay apart by locking their density to the value of the background parameter $k$. The solution of this constraint is however rather different at the two points $g=0$ and $g=\infty$ : for $g=0$, it is the geometric, or kinematic, condition of noncommutativity (39), while at $g=\infty$ this is a dynamical condition set by a repulsive potential with appropriate strength.

- Note also that the $g=\infty$ theory is not, by itself, less difficult than the abinitio quantum Hall problem: the gap is non-perturbative and there are no small parameters. The advantage of embedding the problem into the matrix theory is that of making contact with the solvable $g=0$ limit, as we discuss in the next section.

\subsection{Conjecture on the phase diagram}

In Figure (6) we illustrate the phase diagram of the Maxwell-Chern-Simons matrix theory as a function of its parameters $\mathbf{B} / m$ and $g$. The quantized background charge $\mathbf{B} \theta=k$ is held fixed over the diagram together with average density of the system.

The axes $g=0$ and $g=\infty$ have been discussed in sections 5 and 6.1 , respectively. For $g=0$, the theory is solvable and displays a set of states that are in one-to-one relation with the Laughlin and Jain ground states with filling fractions $\nu=m /(m k+1)$. These 


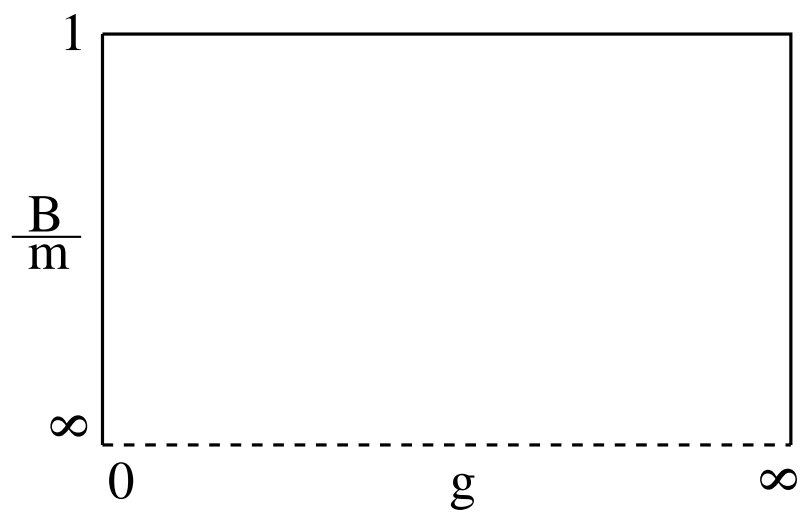

Figure 6. Phase diagram of the Maxwell-Chern-Simons matrix theory. The axes $g=0$ and $g=\infty$ have been discussed in sections 5 and 6 , respectively. The Chern-Simons matrix model sits at the left down corner.

non-degenerate states are selected by choosing the appropriate projection $A^{m} \approx 0$ and the values of $k$ and the density (or the angular momentum). For $g=\infty$, we found that the theory describes a realistic Hall system, but its ground states are difficult to find.

In ref.[12], we conjectured that the matrix ground states at $g=0$ could match one-to-one the phenomenological Jain states that are good ansatz in the physical limit $g=\infty$ (including the case of Calogero interaction) [5][16]: indeed, the two sets of states become identical in the limit of both $\bar{X}, X$ diagonal, that is (classically) achieved at $g=\infty$. In order to prove this conjecture, we would need to consider the evolution of the matrix ground states as the coupling is varied in between, $0<g<\infty$, and to check that the gap never vanishes, i.e. that there are no phase transitions in $(B, g)$ plane separating the $g=0$ and $g=\infty$ regions at the specific density values [12]. This conjecture of smooth evolution of matrix Jain states is indirectly supported by the numerical analyses, showing that the Jain wavefunctions are accurate ground states of the $g=\infty$ theory. Further support is given by the form of the semiclassical density of $g=0$ matrix states that is the qualitatively the same of $g=\infty$ Jain incompressible fluids states.

Let us finally remark that, the limit $\mathbf{B} \rightarrow \infty$ cannot be taken at $g=0$, because quasi-particle excitations and Jain states in the matrix theory have energies of $O(\mathbf{B})$ and would be projected out. Instead, the limit $\mathbf{B}=\infty$ can surely be taken in the $g=\infty$ physical theory (holding $k=\mathbf{B} \theta$ fixed), because the fractional quantum Hall states are known to remain stable. This implies that the two limits are ordered: the correct sequence is $\lim _{\mathbf{B} \rightarrow \infty} \lim _{g \rightarrow \infty} \Psi$, and the opposite choice is cut out in the phase diagram of Fig.6.

\section{Conclusions}

We have reviewed the description of the fractional quantum Hall effect given by gauge matrix theories, that provide one realization of the composite-fermion correspondence. 
In particular, the Maxwell-Chern-Simons theory, supplemented by certain projections of states, reproduces the Jain hierarchical construction of ground state wavefunctions. These results support the idea that the fractional Hall states should be uniquely characterized by algebraic conditions and gauge invariance, rather than by detailed dynamics, because they are exceptionally robust and universal.

The study of the phase diagram of the matrix theory is clearly necessary to make better contact between the nice results $(g=0)$ and the physical regime $(g=\infty)$, upon varying the potential $V=g \operatorname{Tr}[\bar{X}, X]^{2}$. We plan to study the evolution of matrix ground states for $g>0$ by including the quartic potential in the semiclassical analysis within the mean-field approximation.

One point to develop is the study of edge excitations of matrix Jain states [40] and the comparison with the conformal field theory descriptions [20]: in particular, the realization of the $S U(n)$ symmetry, for $\nu=n /(2 k n+1)$, that is still debated [41]. Another open problem is the derivation of the fractional statistics of quasiparticles in the matrix theory setting. Both issues require an improvement of the $A^{n} \approx 0$ projection that could better handle excitations above the ground state.

\section{Acknowledgments}

The authors would like to thank the hospitality of the G. Galilei Institute for Theoretical Physics, Florence. This work was partially funded by the ESF programme INSTANS: Interdisciplinary Statistical and Field Theory Approaches to Nanophysics and Low Dimensional Systems, and by the MUR grant Fisica Statistica dei Sistemi Fortemente Correlati all'Equilibrio e Fuori dall'Equilibrio.

\section{References}

[1] For a review see: R. A. Prange and S. M. Girvin, The Quantum Hall Effect, Springer, Berlin (1990); S. Das Sarma and A. Pinczuk, Perspectives in Quantum Hall effects, Wiley, New York (1997).

[2] D. Tsui, H. Sormer, A .Gossard, Two-Dimensional Magnetotransport in the Extreme Quantum Limit, Phys. Rev. Lett. 48 (1982) 1559.

[3] R. B. Laughlin, Anomalous Quantum Hall Effect: An Incompressible Quantum Fluid with Fractionally Charged Excitations, Phys. Rev. Lett. 50 (1983) 1395; Elementary Theory: the Incompressible Quantum Fluid, in R. A. Prange and S. M. Girvin, Ref. [1].

[4] L. Saminadayar, D. C. Glattli, Y. Jin, and B. Etienne, Observation of the e/3 Fractionally Charged Laughlin Quasiparticle, Phys. Rev. Lett. 79 (1997) 2526; R. de-Picciotto, M. Reznikov, M. Heiblum, V. Umansky, G. Bunin and D. Mahalu, Direct observation of a fractional charge, Nature 389 (1997) 162.

[5] J. Jain, Composite Fermions, Cambridge Univ. Press, Cambridge (2007).

[6] A. Lopez, E. Fradkin, Fractional Quantum Hall Effect and Chern-Simons gauge theories, Phys. Rev. B 44 (1991) 5246; Universal properties of the wave functions of fractional quantum Hall systems, Phys. Rev. Lett. 69 (1992) 2126.

[7] S. Kivelson, D.-H. Lee, and S.-C. Zhang, Global phase diagram in the quantum Hall effect, Phys. Rev. B 46 (1992) 2223; S. C. Zhang, The Chern-Simons-Landau-Ginzburg theory of 
the fractional quantum Hall effect, Int. J. Mod. Phys. B 6 (1992) 25; G. Murthy, R. Shankar, Hamiltonian Theories of the FQHE, Rev. Mod. Phys.75 (2003) 1101.

[8] L. Susskind, The quantum Hall fluid and non-commutative Chern Simons theory, hep-th/0101029.

[9] A. P. Polychronakos, Quantum Hall states as matrix Chern-Simons theory, JHEP 0104 (2001) 011; Quantum Hall states on the cylinder as unitary matrix Chern-Simons theory, JHEP 0106 (2001) 070.

[10] S. Hellerman and M. Van Raamsdonk, Quantum Hall physics equals noncommutative field theory, JHEP 0110 (2001) 039.

[11] A. Cappelli and M. Riccardi, Matrix model description of Laughlin Hall states, J. Stat. Mech. 0505 (2005) P001.

[12] A. Cappelli and I. D. Rodriguez, Jain states in a matrix theory of the quantum Hall effect, JHEP 0612 (2006) 056.

[13] T. H. Hansson, J. Kailasvuori, A. Karlhede and R. von Unge, Solitons and Quasielectrons in the Quantum Hall Matrix Model, Phys. Rev. B 72 (2005) 205317.

[14] A. Cappelli and I. D. Rodriguez, Semiclassical Droplet States in Matrix Quantum Hall Effect, JHEP 0802 (2008) 046.

[15] A. Cappelli, C. A. Trugenberger and G. R. Zemba, Infinite symmetry in the quantum Hall effect, Nucl. Phys. B 396 (1993) 465; Large N limit in the quantum Hall Effect, Phys. Lett. B 306 (1993) 100; for a review, see: Nucl. Phys. B (Proc. Suppl.)33C (1993) 21.

[16] F. D. M. Haldane, The hierarchy of fractional states and numerical studies, in R. A. Prange and S. M. Girvin [1]; J. Jain et al., in [5]; A. Cappelli, C. Méndez, J. Simonin, G. R. Zemba, Numerical study of hierarchical quantum Hall edge states on the disk geometry, Phys. Rev. B 58 (1998) $16291 ;$

[17] F. Wilczek, Fractional Statistics and Anyon Superconductivity, World Scientific, Singapore (1990).

[18] A. Lopez, E. Fradkin, Fermionic Chern-Simons Field Theory for the Fractional Hall Effect, in Composite Fermions: A Unified View of the Quantum Hall Regime, O. Heinonen Ed., World Scientific, Singapore (1998).

[19] X.-G. Wen, Quantum Field Theory of Many-Body Systems, Oxford Univ. Press, Oxford (2004).

[20] J. Fröhlich and A. Zee, Large scale physics of the quantum Hall fluid, Nucl. Phys. B 364 (1991) 517; X.-G. Wen and A. Zee, Classification of Abelian quantum Hall states and matrix formulation of topological fluids, Phys. Rev. B 46 (1993) 2290; A. Cappelli, C. A. Trugenberger and G. R. Zemba, Classification of quantum Hall universality classes by W(1+infinity) symmetry, Phys. Rev. Lett. 72 (1994) 1902; Stable hierarchical quantum hall fluids as W(1+infinity) minimal models, Nucl. Phys. B 448 (1995) 470.

[21] J. H. Brodie, L. Susskind and N. Toumbas, How Bob Laughlin tamed the giant graviton from Taub-NUT space, JHEP 0102 (2001) 003; B. Freivogel, L. Susskind and N. Toumbas, A two fluid description of the quantum Hall soliton, hep-th/0108076; O. Bergman, Y. Okawa and J. H. Brodie, The stringy quantum Hall fluid, JHEP 0111 (2001) 019; A. Ghodsi, A. E. Mosaffa, O. Saremi and M. M. Sheikh-Jabbari, LLL vs. LLM: Half BPS sector of $N=4 S Y M$ equals to quantum Hall system, Nucl. Phys. B 729 (2005) 467; J. Dai, X. J. Wang and Y. S. Wu, Dynamics of giant-gravitons in the LLM geometry and the fractional quantum Hall effect, Nucl. Phys. B 731 (2005) 285.

[22] G. V. Dunne, R. Jackiw and C. A. Trugenberger, 'Topological' (Chern-Simons) Quantum Mechanics, Phys. Rev. D 41 (1990) 661.

[23] R. Jackiw, S. Y. Pi and A. P. Polychronakos, Noncommuting gauge fields as a Lagrange fluid, Ann. Phys. (NY) 301 (2002) 157; R. Jackiw, A. P. Polychronakos, Perfect fluid theory and its extensions, J. Phys. A 37327 (2004); A. P. Polychronakos, Noncommutative Fluids, arXiv:0706.1095, to appear in Seminaire Poincare X, IHP, Paris.

[24] N. Seiberg and E. Witten, String theory and noncommutative geometry, JHEP 9909 (1999) 032; M. R. Douglas and N. A. Nekrasov, Noncommutative field theory, Rev. Mod. Phys. 73 (2001) 977. 
[25] N. Seiberg, A note on background independence in noncommutative gauge theories, matrix model and tachyon condensation, JHEP 0009 (2000) 003; J. H. Brodie, L. Susskind and N. Toumbas, in $[21]$.

[26] J. Hoppe, Ph.D. thesis (Massachusetts Institute of Technology), (1982).

[27] V. P. Nair and A. P. Polychronakos, On level quantization for the noncommutative Chern-Simons theory, Phys. Rev. Lett. 87 (2001) 030403.

[28] B. Morariu and A. P. Polychronakos, Finite noncommutative Chern-Simons with a Wilson line and the quantum Hall effect, JHEP 0107 (2001) 006.

[29] D. Karabali and B. Sakita, Orthogonal basis for the energy eigenfunctions of the Chern-Simons matrix model, Phys. Rev. B 65 (2002) 075304; Chern-Simons matrix model: Coherent states and relation to Laughlin wavefunctions, Phys. Rev. B 64 (2001) 245316.

[30] T. H. Hansson and A. Karlhede, Charges and Currents in the Noncommutative Chern-Simons Theory of the QHE, cond-mat/0109413; T. H. Hansson, J. Kailasvuori and A. Karlhede, Charge and Current in the Quantum Hall Matrix Model, Phys. Rev. B 68, 035327 (2003).

[31] E. Brezin, C. Itzykson, G. Parisi and J. B. Zuber, Planar Diagrams, Commun. Math. Phys. 59 (1978) 35; M.L. Mehta, Random Matrices and the statistical theory of energy levels, Academic Press, New York (1967).

[32] J. A. Harvey, Komaba lectures on noncommutative solitons and D-branes, [hep-th/0102076]; J. Polchinski, String Theory, Vol.2 Cambridge Univ. Press, Cambridge (1998).

[33] T. Banks, W. Fischler, S. H. Shenker and L. Susskind, M theory as a matrix model: A conjecture, Phys. Rev. D 55 (1997) 5112; W. Taylor, M(atrix) theory: Matrix quantum mechanics as a fundamental theory, Rev. Mod. Phys. 73 (2001) 419.

[34] E. Witten, Bound states of strings and p-branes, Nucl. Phys. B 460 (1996) 335.

[35] J. H. Park, On a matrix model of level structure, Class. Quant. Grav. 19 (2002) L11.

[36] V. Pasquier, F.D.M. Haldane, A dipole interpretation of the $\nu=1 / 2$ state, Nucl. Phys. B (FS) 516 (1998) 719; V. Pasquier, Skyrmions in the quantum Hall effect and noncommutative solitons, Phys. Lett. B 490 (2000) 258.

[37] J. Lambert and M. B. Paranjape, Quasi-hole solutions in finite noncommutative Maxwell-ChernSimons theory, JHEP 0705 (2007) 007.

[38] A. P. Polychronakos, Multidimensional Calogero systems from matrix models, Phys. Lett. B 408 (1997) 117.

[39] A. P. Polychronakos, Physics and mathematics of Calogero particles, J. Phys. A 39 (2006) 12793.

[40] D. C. Cabra, N. E. Grandi, Incidence of the boundary shape in the effective theory of fractional quantum Hall edges, Phys. Rev. B 77 (2008) 115107; I. D. Rodriguez, Edge excitations of the Chern Simons matrix theory for the FQHE, arXiv:0812.4531.

[41] A. Cappelli and G. R. Zemba, Hamiltonian formulation of the W(1+infinity) minimal models, Nucl. Phys. B 540 (1999) 610; D. Orgad and O. Agam, Correlated Tunneling and the Instability of the Fractional Quantum Hall Edge Phys. Rev. Lett. 100 (2008) 156802. 